TRANSACTIONS OF THE

AMERICAN MATHEMATICAL SOCIETY

Volume 363, Number 3, March 2011, Pages 1365-1397

S 0002-9947(2010)04987-6

Article electronically published on October 22, 2010

\title{
HETEROCLINIC TRAVELLING WAVES OF GRADIENT DIFFUSION SYSTEMS
}

\author{
NICHOLAS D. ALIKAKOS AND NIKOLAOS I. KATZOURAKIS
}

\begin{abstract}
We establish the existence of travelling waves to the gradient system $u_{t}=u_{z z}-\nabla W(u)$ connecting two minima of $W$ when $u: \mathbb{R} \times(0, \infty) \longrightarrow$ $\mathbb{R}^{N}$; that is, we establish the existence of a pair $(U, c) \in\left[C^{2}(\mathbb{R})\right]^{N} \times(0, \infty)$, satisfying

$$
\left\{\begin{array}{l}
U_{x x}-\nabla W(U)=-c U_{x} \\
U( \pm \infty)=a^{ \pm}
\end{array}\right.
$$

where $a^{ \pm}$are local minima of the potential $W \in C_{\mathrm{loc}}^{2}\left(\mathbb{R}^{N}\right)$ with $W\left(a^{-}\right)<$ $W\left(a^{+}\right)=0$ and $N \geq 1$. Our method is variational and based on the minimization of the functional $E_{c}(U)=\int_{\mathbb{R}}\left\{\frac{1}{2}\left|U_{x}\right|^{2}+W(U)\right\} e^{c x} d x$ in the appropriate space setup. Following Alikakos and Fusco (2008), we introduce an artificial constraint to restore compactness and force the desired asymptotic behavior, which we later remove. We provide variational characterizations of the travelling wave and the speed.
\end{abstract}

\section{INTRODUCTION}

Assume we are given a potential $W \in C_{\text {loc }}^{2}\left(\mathbb{R}^{N}\right)$ with several local minima, in general at different levels. Let $a^{+}, a^{-}$be local minima with $W\left(a^{+}\right)=0, W\left(a^{-}\right)<0$. We consider the problem of existence of a solution $(U, c)$ to the system

$$
\left\{\begin{array}{l}
U_{x x}-\nabla W(U)=-c U_{x} \\
U( \pm \infty)=a^{ \pm}
\end{array}\right.
$$

where $c>0$ and $U: \mathbb{R} \longrightarrow \mathbb{R}^{N}$ is in $\left[C^{2}(\mathbb{R})\right]^{N}$ connecting $a^{ \pm}$, the dimension being any $N \geq 1$. A typical potential with two minima and $N=2$ is shown in Figure 1. The solutions of problem (11) are known as heteroclinic travelling waves. They are special solutions of the form $U(z-c t)=u(z, t)$ to the diffusion system with gradient structure:

$$
u_{t}=u_{z z}-\nabla W(u), \quad u=u(z, t): \quad \mathbb{R} \times(0, \infty) \longrightarrow \mathbb{R}^{N},
$$

and, in addition, heteroclinic connections of the dynamical system corresponding to the 2nd order ODE system $U_{x x}-\nabla W(U)=-c U_{x}$. Physically, problem (11) can be interpreted as the Newtonian Law of motion with force term $-\nabla(-W)$ due to the potential $-W$ and dissipation (friction) term $-c U_{x}$. In this context, $U(x)$

Received by the editors December 24, 2007 and, in revised form, November 30, 2008.

2010 Mathematics Subject Classification. Primary 35C07.

Key words and phrases. Gradient diffusion systems, parabolic PDEs, travelling waves, heteroclinic connections.

The first author was partially supported by Kapodistrias Grant No. 70/4/5622 at the University of Athens.

(C)2010 American Mathematical Society Reverts to public domain 28 years from publication 

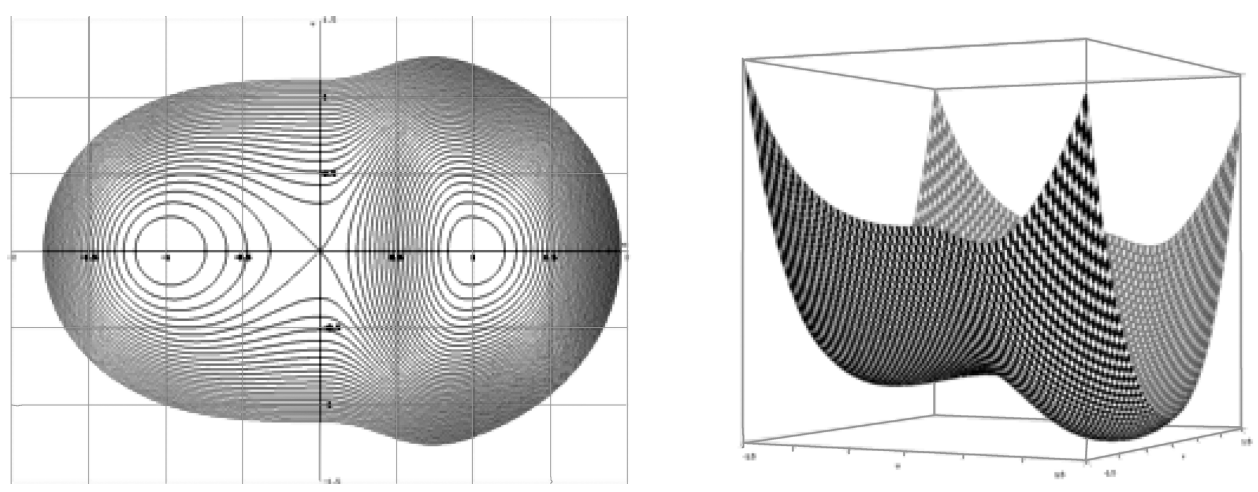

Figure 1. Simulation of the standard 2-well $W$ deformed (Example 35), having minima at different levels.

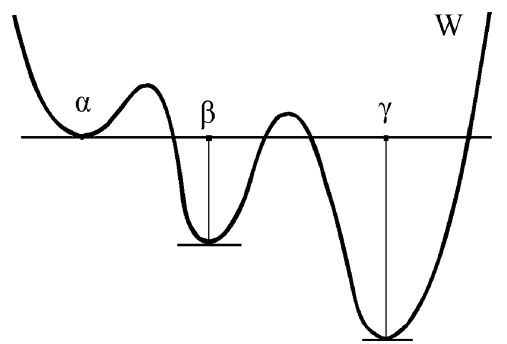

FIGURE 2. In general, no $\alpha-\gamma$ connection exists.

represents the trajectory of an ideal unit mass particle going from a global maximum to another local maximum of $-W$, asymptotically in time. Problem (11) with $c=0$ is a special case known as the "standing wave" heteroclinic connection problem. It reduces to a Hamiltonian system $U_{x x}=\nabla W(U)$ for a potential with minima at the same level. This case for general $N>1$ has been studied by Sternberg in St, Alikakos-Fusco in $\mathrm{A}-\mathrm{F}$ and in great detail for $N=2$ by Alikakos, Betelú, and Chen in $\mathrm{A}-\mathrm{Be}-\mathrm{C}]$.

The scalar case $N=1$ and $c>0$ of (11) is textbook material from the viewpoint of existence (e.g., $\mathrm{He}$ p. 128, $\mathrm{Ev}$, p. 175). The global stability of the connection for the scalar case of (2) has been studied in the classical papers of Fife and McLeod F-McL, F-McL2 and recently by Gallay and Risler in G-R. Already in the scalar case, existence for (1) of a heteroclinic between two minima is not always guaranteed in the presence of a third one, as has been observed in [F-McL] (Figure 2).

In the vector case $N>1$ and $c \neq 0$ for (1), maximum and comparison principles are no longer available and as a result only special systems have been studied. We refer to the monograph of the Volperts [V] for monotone systems and numerous related references.

In a very recent paper, E. Risler $[\mathbb{R}$ has established the existence of solutions to (11), as a by-product of his study of the parabolic semiflow of (2). Among other results, Risler studies the case of a bistable potential and proves the existence of a travelling wave connecting the global minimum of $W$ with a local minimum, 
as in the present paper. However, our hypotheses are generally different, and in particular our nondegeneracy assumption on the minima of $W$ is very weak (see (h*) in $\S 6$, and ( $\left.\mathrm{h}^{* *}\right)$ in $\left.\S 8\right)$.

Another very recent paper that establishes existence of travelling waves, actually for a generalization of (1), is that of Lucia-Muratov-Novaga [LMN]. Their method has similarities with ours, but their hypotheses are different and not directly addressing the potential $W$.

In the present paper we choose to work directly with the time-independent problem (11). We prove the existence of heteroclinic travelling waves for potentials with several minima under weak coercivity requirements which allow for potentials unbounded from below. We establish connections between possibly degenerate minima, imposing assumptions only on the geometry of the sublevel set $\{W \leq \alpha\} \subseteq \mathbb{R}^{N}$ for $\alpha>0$ small, which encloses the minima (assumptions (h*) in Section 6, (h**) in Section 8).

Our approach is variational: we introduce a weighted action functional, already introduced in Fife-McLeod ([F-McL, [F-McL2] $)$, to obtain travelling wave solutions to (11) as (local) minimizers of the weighted action

$$
E_{c}(U)=\int_{\mathbb{R}}\left\{\frac{1}{2}\left|U_{x}\right|^{2}+W(U)\right\} e^{c x} d x
$$

in the Fréchet space of vector functions $\left[H_{\mathrm{loc}}^{1}(\mathbb{R})\right]^{N}$, utilizing certain devices to overcome the unboundedness and compactness problems of $E_{c}$. We show that action-minimizing travelling waves $(U, c)$ are characterized by the property $E_{c}(U)=$ 0 , and they can be derived as solutions to

$$
E_{c}(U)=\inf \left\{E_{c}(V): V \in\left[H_{\mathrm{loc}}^{1}(\mathbb{R})\right]^{N}, V( \pm \infty)=a^{ \pm}\right\}, \quad E_{c}(U)=0 .
$$

(44) is originally due to Muratov [M]. Certain other ideas of his are also utilized in the present work in the determination of $c$ (Propositions 25, 30).

We now give a brief description of our method. A formal computation shows that critical points of $E_{c}$ correspond to weak solutions of (11). We wish to construct solutions of $U_{x x}-\nabla W(U)=-c U_{x}$, with the desired behavior at infinity $U( \pm \infty)=a^{ \pm}$, by minimizing (3), in the appropriate setup. Minimization cannot be done directly, because the unbounded domain $\mathbb{R}$ excludes compactness in all reasonable functional spaces, while the asymptotic behavior required in (11) cannot be guaranteed.

In addition, (3) is unbounded from below for certain values of $c>0$, a difficulty not present when $c=0$, and moreover it is sensitive to translations: $E_{c}(U(\cdot-\delta))=$ $e^{c \delta} E_{c}(U)$. Thus, a minimizing sequence may converge to the trivial minimizers $a^{ \pm}$ with $E_{c}\left(a^{+}\right)=0, E_{c}\left(a^{-}\right)=-\infty$.

To overcome these problems, we first solve a constrained minimization problem, utilizing the unilateral constraint method introduced by Alikakos and Fusco in $\mathrm{A}-\mathrm{F}$ : we fix two arbitrary parameters $c, L>0$ and we minimize $E_{c}$ directly within the admissible set of functions in $\left[H_{\mathrm{loc}}^{1}(\mathbb{R})\right]^{N}$ whose graph lies in the cylinders $(-\infty,-L] \times \mathbb{B}\left(a^{-}, r_{0}\right)$ and $[L,+\infty) \times \mathbb{B}\left(a^{+}, r_{0}\right)$ enclosing the two minima $a^{ \pm}$ to be connected. Minimization leads to a 2-parameter family of minimizers in $c, L>0$. Then $L$ is increased until the constraint is not realized for some minimizer, thus solving the Euler-Lagrange equation (1) for some specific value of the other parameter $c=c^{*}>0$. 
This device bounds (3) from below and allows us to "capture" an object which is close to a solution to (11). Constrained minimizers are piecewise solutions (except possibly at the rims $\left.\{ \pm L\} \times \partial\left(\mathbb{B}\left(a^{ \pm}, r_{0}\right)\right)\right)$ converging asymptotically to $a^{ \pm}$, for all $c>0$. The main effort in the proof is devoted to showing that the constraint is in fact not realized for a specific $c^{*}>0$ and for sufficiently large $L$.

The role of " $c$ " is as follows. We incorporate into $E_{c}$ an arbitrary parameter $c>0$ which, until Section 6 , is always arbitrary and fixed. In particular, we do not view $c$ as a functional $c(U)$ of $U$. The specific $c=c^{*}$ which guarantees existence is determined by the requirement that $E_{c^{*}}\left(U_{L}\right)=0$ for sufficiently large $L \geq L^{*}$. This is necessary for the existence of minimizers since, as observed by Muratov [M], translation sensitivity of (3) shows that the only possible finite infimum of (3) is zero.

The paper is organized as follows. In Section 2 we solve the constrained problem for $E_{c}$ in $\left[H_{\mathrm{loc}}^{1}(\mathbb{R})\right]^{N}$, resulting in a 2-parameter family of minimizers in $c>0$ and $L>0$. In Section 3, we follow $[\mathrm{A}-\mathrm{F}$ in assuming a very mild nondegeneracy (h) at the minima $a^{ \pm}$, and show that constrained minimizers are piecewise solutions to $U_{x x}-\nabla W(U)=-c U_{x}$, solving it on $\mathbb{R} \backslash\{ \pm L\}$ and converging to $a^{ \pm}$at $\pm \infty$.

In Section 4 we introduce the main tool for removing the constraint: two replacement lemmas, modeled after Lemmas 3.3, 3.4 in $\mathrm{A}-\mathrm{F}$. The new ingredient is the introduction of a convex set in the place of a ball, which allows controlling the solution far from the minima. The presentation here is self-contained and independent of the rest of the paper.

In Section 5 we establish certain energy identities. In particular, they imply an energy equipartition at $+\infty$ and that $E_{c}\left(U_{L}\right)$ measures the jumps $\left.\left[\left[\left(U_{L}\right)_{x}\right]\right]\right|_{ \pm L}$.

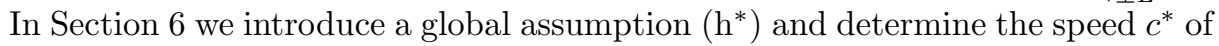
the travelling wave. $c^{*}$ is defined by means of a variational formula (see (27) ) which is similar to a formula of Heinze Hei . Utilizing tools from Sections 4, 5, we prove that $c^{*}$ satisfies the desired properties (Proposition 25). Hence, we distinguish the suitable $E_{c^{*}}$ among all $\left\{E_{c}: c>0\right\}$. The variational formulation (4) which implies existence for (1) is also given here.

In Section 7 we prove the existence of a solution by removing the constraint and deriving explicit bounds on $c^{*} \in\left[c_{\min }, c_{\max }\right]$, by means of our variational formulation (4).

In Section 8 we show that the assumption (h*) can be relaxed to include potentials that are unbounded from below or have other critical points besides $a^{ \pm}$(cf. A-F]). Finally, in the Appendix we discuss the optimality of our assumptions.

Our proof includes the special case $W\left(a^{-}\right)=W\left(a^{+}\right)=0, c=0$ that was treated in $\mathrm{A}-\mathrm{F}$.

\section{The CONSTRAined Minimization PROBLEM}

Here we solve a minimization problem for $E_{c}(U)=\int_{\mathbb{R}}\left\{\frac{1}{2}\left|U_{x}\right|^{2}+W(U)\right\} e^{c x} d x$ in the local Sobolev space $\left[H_{\text {loc }}^{1}(\mathbb{R})\right]^{N}$ of the vector $U: \mathbb{R} \longrightarrow \mathbb{R}^{N}$. $\left[H_{\text {loc }}^{1}(\mathbb{R})\right]^{N}$ admits a Fréchet topology, defined by the seminorms of $\left[H^{1}(-m, m)\right]^{N}, m \geq 1$. Technically, instead of $\left[H_{\mathrm{loc}}^{1}(\mathbb{R})\right]^{N}$ we use its isomorphic copy $\left[H_{\mathrm{loc}}^{1}\left(\mathbb{R}, e^{\text {cId }}\right)\right]^{N}$ with weight $x \mapsto e^{c x}$, the standard Lebesgue measure $d x$ being replaced by the absolutely continuous $e^{c x} d x$. It is only a matter of convenience, since minimization gives derivatives bounded in $\left[L^{2}\left(\mathbb{R}, e^{c I d}\right)\right]^{N} .\left[C_{\mathrm{loc}}^{k}(\mathbb{R})\right]^{N}$ will denote the space of $C^{k}$ vector functions equipped with the Fréchet topology of uniform convergence together with 
all the derivatives over compact sets, while $\left[C^{k}(\mathbb{R})\right]^{N}$ denotes the space of bounded $C^{k}$ vector functions with its standard norm. We shall frequently decompose $W$ as $W=W^{+}-W^{-}$, where $W^{+}=\max \{W, 0\}$ and $W^{-}=\max \{-W, 0\}$.

Lemma 1 (Characterization of the speed). Assume that a solution $(U, c)$ to (1) exists, satisfying $U_{x}( \pm \infty)=0$ up to subsequences. Then:

$$
W^{-}\left(a^{-}\right)=c \int_{\mathbb{R}}\left|U_{x}\right|^{2} d x \quad \text { and } \quad c\left(a^{+}-a^{-}\right)=\int_{\mathbb{R}} \nabla W(U) d x .
$$

Proof of Lemma 1. The equation readily implies $-U_{x x} \cdot U_{x}+\nabla W(U) \cdot U_{x}=c\left|U_{x}\right|^{2}$. Hence,

$$
\begin{aligned}
c \int_{\mathbb{R}}\left|U_{x}\right|^{2} d x & =-\int_{\mathbb{R}}\left(\frac{1}{2}\left|U_{x}\right|^{2}\right)_{x} d x+\int_{\mathbb{R}}(W(U))_{x} d x \\
& = \pm 0+W(U(+\infty))-W(U(-\infty)) \\
& =-W\left(a^{-}\right) .
\end{aligned}
$$

Moreover, again from the equation we have

$$
\begin{aligned}
\int_{\mathbb{R}} \nabla W(U) d x & =\int_{\mathbb{R}}\left(U_{x x}+c U_{x}\right) d x \\
& =0-0+c\left(a^{+}-a^{-}\right) .
\end{aligned}
$$

As a consequence of Lemma 1, if $U( \pm \infty)=a^{ \pm}$and $W\left(a^{+}\right)=0>W\left(a^{-}\right)$, then $c$ must be positive.

Now take $L>0$ and $r_{0}>0$ small, such that $W(u) \geq 0$ for $\left|a^{+}-u\right| \leq r_{0}$ and $W(u)<0$ for $\left|a^{-}-u\right| \leq r_{0}$. We introduce the constraint sets:

$$
\begin{aligned}
& \mathcal{X}_{L}^{+}:=\left\{U \in\left[H_{\mathrm{loc}}^{1}\left(\mathbb{R}, e^{c \mathrm{Id}}\right)\right]^{N}:\left|U(x)-a^{+}\right| \leq r_{0}, x \geq+L\right\}, \\
& \mathcal{X}_{L}^{-}:=\left\{U \in\left[H_{\mathrm{loc}}^{1}\left(\mathbb{R}, e^{c \mathrm{Id}}\right)\right]^{N}:\left|U(x)-a^{-}\right| \leq r_{0}, x \leq-L\right\},
\end{aligned}
$$

and set $\mathcal{X}_{L}:=X_{L}^{+} \cap \mathcal{X}_{L}^{-}$. Pointwise values make sense by means of the imbedding $\left[H_{\text {loc }}^{1}\left(\mathbb{R}, e^{c \mathrm{Id}}\right)\right]^{N} \hookrightarrow\left[C_{\mathrm{loc}}^{0}(\mathbb{R})\right]^{N}$.

Theorem 2 (Existence of constrained minimizers). Let $W$ be a potential in $C_{\text {loc }}^{2}\left(\mathbb{R}^{N}\right)$ and let $a^{ \pm}$be two of its local minima, with $W\left(a^{-}\right)<0=W\left(a^{+}\right)$, and $a^{-}$its global minimum. We assume that $W^{-1}\left(\left[W\left(a^{-}\right), 0\right]\right)$ is compact in $\mathbb{R}^{N}$. If $L>0, c>0$ are fixed parameters, then the minimization problem

$$
E_{c}\left(U_{L}\right)=\inf _{\mathcal{X}_{L}}\left\{E_{c}\right\}
$$

has a solution $U_{L}$ in $\mathcal{X}_{L} \subseteq\left[H_{\mathrm{loc}}^{1}\left(\mathbb{R}, e^{c \mathrm{Id}}\right)\right]^{N}$.

The assumption on $W$ implies $\liminf _{|u| \rightarrow \infty}[W(u)] \geq 0$. This will be relaxed eventually, allowing for potentials with several local minima and possibly unbounded negative values, by means of a localization. We denote the minimizers of $E_{c}$ into $\mathcal{X}_{L}$ by $U_{L}$ instead of the more accurate notation $U_{c, L}$, suppressing the dependence on the parameter $c>0$ which (until Section 6) is always fixed.

Proof of Theorem 2. We first show that $\mathcal{X}_{L} \neq \emptyset$ together with $-\infty<\inf \mathcal{X}_{L}\left\{E_{c}\right\}<$ $\infty$. Since we are interested only in increasing the parameter $L$ later, we restrict, as we can, our attention to $L \geq 1$. 


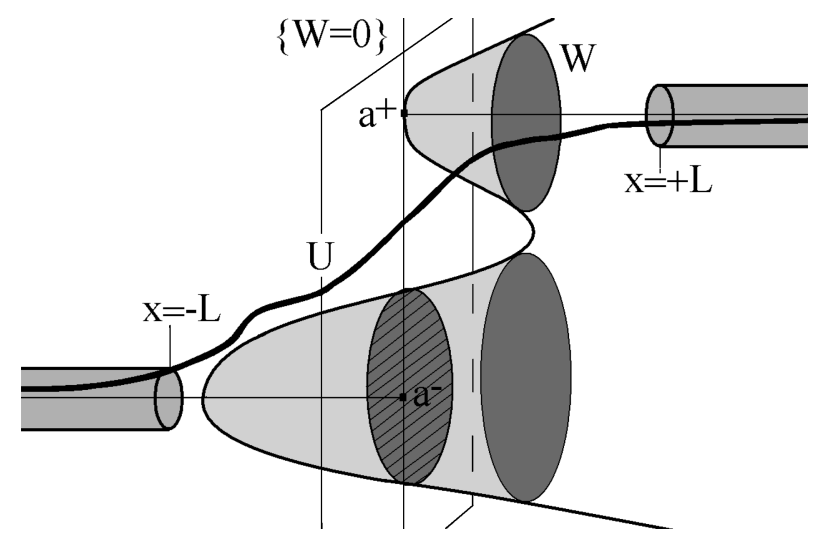

FiguRE 3. The device of constrained minimization which restores compactness and boundedness.

Claim. There exists an affine function $U_{\text {aff }} \in \mathcal{X}_{L} \cap\left[W_{\text {loc }}^{1, \infty}(\mathbb{R})\right]^{N}$ such that

$$
-\infty<-\frac{e^{c L} W^{-}\left(a^{-}\right)}{c} \leq \inf _{\mathcal{X}_{L}}\left\{E_{c}\right\} \leq E_{c}\left(U_{\text {aff }}\right)<\infty .
$$

Proof of Claim. Let $\chi_{A}$ denote the characteristic of $A \subseteq \mathbb{R}$. We set

$$
U_{\mathrm{aff}}(x):=a^{-} \chi_{(-\infty,-1)}+\left(\frac{1-x}{2} a^{-}+\frac{1+x}{2} a^{+}\right) \chi_{[-1,1]}+a^{+} \chi_{(1, \infty)} .
$$

Clearly, $\left(U_{\mathrm{aff}}\right)_{x} \in\left[L_{\mathrm{loc}}^{\infty}(\mathbb{R})\right]^{N}$ and exists a.e. on $\mathbb{R}$. Thus, $U_{\mathrm{aff}} \in\left[H_{\mathrm{loc}}^{1}\left(\mathbb{R}, e^{\text {cId }}\right)\right]^{N}$. We calculate

$$
\begin{aligned}
E_{c}\left(U_{\mathrm{aff}}\right)= & \int_{-\infty}^{-1}\left(0+W\left(a^{-}\right)\right) e^{c x} d x+\int_{1}^{\infty}\left(0+W\left(a^{+}\right)\right) e^{c x} d x \\
& +\int_{-1}^{1}\left\{\frac{1}{2}\left|\frac{a^{+}-a^{-}}{2}\right|^{2}+W\left(\frac{1-x}{2} a^{-}+\frac{1+x}{2} a^{+}\right)\right\} e^{c x} d x \\
\leq & \int_{-1}^{1}\left\{\frac{1}{2}\left|\frac{a^{+}-a^{-}}{2}\right|^{2}+W^{+}\left(\frac{1-x}{2} a^{-}+\frac{1+x}{2} a^{+}\right)\right\} e^{c x} d x \\
& +\frac{1}{c} e^{-c} W\left(a^{-}\right) .
\end{aligned}
$$

Hence, if we set $E_{c}^{+}(U):=\int_{\mathbb{R}}\left\{\frac{1}{2}\left|U_{x}\right|^{2}+W^{+}(U)\right\} e^{c x} d x$, we obtain

$$
E_{c}\left(U_{\mathrm{aff}}\right) \leq-e^{-c} \frac{W^{-}\left(a^{-}\right)}{c}+e^{c} E_{0}^{+}\left(U_{\mathrm{aff}}\right) .
$$

This implies the upper bound $\sup _{L \geq 1} \inf _{\mathcal{X}_{L}}\left\{E_{c}\right\} \leq \sup _{L \geq 1} E_{c}\left(U_{\text {aff }}\right)<\infty$. If $U$ lies in $\mathcal{X}_{L}$, we have $W^{-}(U(x))=0$ for $x \geq L$ and $W^{+}(U(x))=0$ for $x \leq-L$. Hence, 
for any such $U$, utilizing that $W^{-}(U) \leq W^{-}\left(a^{-}\right)$, we have

$$
\begin{aligned}
E_{c}(U) & =\int_{\mathbb{R}}\left\{\frac{1}{2}\left|U_{x}\right|^{2}+W(U)\right\} e^{c x} d x \\
& =\frac{1}{2} \int_{\mathbb{R}}\left|U_{x}\right|^{2} e^{c x} d x+\int_{\mathbb{R}} W^{+}(U) e^{c x} d x-\int_{\mathbb{R}} W^{-}(U) e^{c x} d x \\
& \geq-\int_{\mathbb{R}} W^{-}(U) e^{c x} d x \\
& \geq-W^{-}\left(a^{-}\right) \int_{-\infty}^{L} e^{c x} d x=-\frac{W^{-}\left(a^{-}\right)}{c} e^{c L} .
\end{aligned}
$$

By $C^{2}$ regularity of the solutions to (1), we may assume that inf $\mathcal{X}_{L}\left[E_{c}\right]<E_{c}\left(U_{\text {aff }}\right)$ strictly. We choose a minimizing sequence $\left\{U_{L}^{n}\right\}_{n \geq 1}$ in $\left[H_{\text {loc }}^{1}\left(\mathbb{R}, e^{c \text { Id }}\right)\right]^{N}$ such that $E_{c}\left(U_{L}^{n}\right) \longrightarrow \inf _{\mathcal{X}_{L}}\left\{E_{c}\right\}$, as $n \rightarrow \infty$. The constraints immediately yield

$$
\left|U_{L}^{n}(x)\right| \leq \max \left\{\left|a^{+}\right|,\left|a^{-}\right|\right\}+r_{0}, \quad x \in(-\infty,-L] \cup[L, \infty) .
$$

Claim (Uniform bounds). There exists a $C=C(c, L, W)>0$ such that

$$
\sup _{n \geq 1}\left\|\left(U_{L}^{n}\right)_{x}\right\|_{\left[L^{2}\left(\mathbb{R}, e^{c I d}\right)\right]^{N}} \leq C, \sup _{n \geq 1}\left\|U_{L}^{n}\right\|_{\left[L^{\infty}(\mathbb{R})\right]^{N}} \leq C .
$$

Proof of Claim. For any $x \in[-L, L]$, we have the estimates

$$
\begin{aligned}
\left|U_{L}^{n}(x)\right| & \leq\left|U_{L}^{n}(-L)\right|+\int_{-L}^{x}\left|\left(U_{L}^{n}\right)_{t}\right| e^{\frac{c t}{2}} e^{\frac{-c t}{2}} d t \\
\leq \max \left\{\left|a^{+}\right|,\left|a^{-}\right|\right\} & +r_{0}+\left(\int_{-L}^{L} e^{-c t} d t\right)^{\frac{1}{2}}\left(\int_{-L}^{x}\left|\left(U_{L}^{n}\right)_{t}\right|^{2} e^{c t} d t\right)^{\frac{1}{2}}, \\
\frac{1}{2} \int_{\mathbb{R}}\left|\left(U_{L}^{n}\right)_{x}\right|^{2} e^{c x} d x & \leq E_{c}\left(U_{\text {aff }}\right)-\int_{\mathbb{R}} W\left(U_{L}^{n}\right) e^{c x} d x \\
& \leq E_{c}\left(U_{\text {aff }}\right)-\int_{\mathbb{R}} W^{+}\left(U_{L}^{n}\right) e^{c x} d x+\int_{\mathbb{R}} W^{-}\left(U_{L}^{n}\right) e^{c x} d x \\
& \leq E_{c}\left(U_{\text {aff }}\right)+\int_{-\infty}^{L} W^{-}\left(U_{L}^{n}\right) e^{c x} d x \\
& \leq E_{c}\left(U_{\text {aff }}\right)+\frac{W^{-}\left(a^{-}\right)}{c} e^{c L} .
\end{aligned}
$$

We conclude:

$$
\frac{1}{2}\left\|\left(U_{L}^{n}\right)_{x}\right\|_{\left[L^{2}\left(\mathbb{R}, e^{c \text { cId }}\right)\right]^{N}}^{2} \leq \frac{W^{-}\left(a^{-}\right)}{c} e^{c L}+E_{c}\left(U_{\text {aff }}\right) .
$$

Utilizing that $\left|U_{L}^{n}(x)\right| \leq \max \left\{\left|a^{+}\right|,\left|a^{-}\right|\right\}+r_{0}$ for $x \in(-\infty,-L] \cup[L, \infty)$, we get

$$
\left\|U_{L}^{n}\right\|_{\left[L^{\infty}(\mathbb{R})\right]^{N}} \leq \max \left\{\left|a^{+}\right|,\left|a^{-}\right|\right\}+r_{0}+\left(\frac{e^{c L}-e^{-c L}}{c}\right)^{\frac{1}{2}}\left\|\left(U_{L}^{n}\right)_{x}\right\|_{\left[L^{2}\left(\mathbb{R}, e^{c I d}\right)\right]^{N}}
$$

We may now proceed to the existence of the minimizer. By the claim above, $\left(U_{L}^{n}\right)_{1}^{\infty}$ is bounded in the locally convex sense in $\left[H_{\mathrm{loc}}^{1}\left(\mathbb{R}, e^{c \mathrm{Id}}\right)\right]^{N}$, with the derivatives bounded in $\left[L^{2}\left(\mathbb{R}, e^{c I d}\right)\right]^{N}$ :

$$
\begin{aligned}
& \sup _{n \geq 1}\left\|U_{L}^{n}\right\|_{\left(H^{1}\left(I, e^{c I d}\right)\right]^{N}} \leq C(c, L, W, I) \text { for all } I \subset \subset \mathbb{R}, \\
& \sup _{n \geq 1}\left\|\left(U_{L}^{n}\right)_{x}\right\|_{\left[L^{2}\left(\mathbb{R}, e^{c \text { cd }}\right)\right]^{N}} \leq C(c, L, W) .
\end{aligned}
$$


By standard compactness arguments, there exists a $U_{L} \in\left[H_{\text {loc }}^{1}\left(\mathbb{R}, e^{c I d}\right)\right]^{N}$ such that, up to a certain subsequence, $U_{L}^{n} \longrightarrow U_{L}$ as $n \rightarrow \infty$ weakly in $\left[H_{\text {loc }}^{1}(\mathbb{R})\right]^{N}$ and $U_{L}^{n} \longrightarrow U_{L}$ in $\left[L_{\text {loc }}^{2}\left(\mathbb{R}, e^{c \mathrm{Id}}\right)\right]^{N}$ and a.e. on $\mathbb{R}$. By weak LSC of the weighted $L^{2}$ norm and the Fatou Lemma for $W\left(U_{L}^{n}\right)+W^{-}\left(a^{-}\right) \chi_{(-\infty, L]} \geq 01$, we have

$$
\begin{gathered}
\int_{\mathbb{R}} \frac{1}{2}\left|\left(U_{L}\right)_{x}\right|^{2} e^{c x} d x \leq \liminf _{n \rightarrow \infty} \int_{\mathbb{R}} \frac{1}{2}\left|\left(U_{L}^{n}\right)_{x}\right|^{2} e^{c x} d x, \\
\int_{\mathbb{R}}\left\{W\left(U_{L}\right)+W^{-}\left(a^{-}\right) \chi_{(-\infty, L]}\right\} e^{c x} d x \\
\leq \liminf _{n \rightarrow \infty} \int_{\mathbb{R}}\left\{W\left(U_{L}^{n}\right)+W^{-}\left(a^{-}\right) \chi_{(-\infty, L]}\right\} e^{c x} d x .
\end{gathered}
$$

Hence, the theorem follows together with the bounds

$$
\begin{aligned}
-\frac{e^{c L} W^{-}\left(a^{-}\right)}{c} \leq E_{c}\left(U_{L}\right) & \leq \lim _{n \rightarrow \infty} E_{c}\left(U_{L}^{n}\right) \\
& \leq-e^{-c} \frac{W^{-}\left(a^{-}\right)}{c}+e^{c} E_{0}^{+}\left(U_{\text {aff }}\right) .
\end{aligned}
$$

\section{Constrained Minimizers ARE PIECEWISE SOlutions}

We will now prove that the constrained minimizers $U_{L}$ of Theorem 2 are piecewise solutions in $\left[C_{\text {loc }}^{2}(\mathbb{R} \backslash\{-L, L\}]^{N}\right.$, while the graphs of $\left.U_{L}\right|_{(-\infty,-L]}$ and $\left.U_{L}\right|_{[L, \infty)}$ are inside the cylinders and converge asymptotically to $a^{ \pm}$. Following $[\mathrm{A}-\mathrm{F}$, we introduce the local monotonicity assumption:

There exists an $R_{0}>0$ such that the map $r \mapsto W\left(a^{ \pm}+r \xi\right)$ has a strictly positive derivative for every $r \in\left(0, R_{0}\right)$ and every $\xi \in \mathbb{R}^{N},|\xi|=1$.

This is a rather weak nondegeneracy assumption, allowing for potentials with degenerate $C^{\infty}$ - flat minima. From now on we assume, as we can, that $r_{0}<R_{0}$; hence $\mathbb{B}\left(a^{ \pm}, r_{0}\right)$ are in the monotonicity region. We will need to express $U_{L}$ in polar form: for any $U$ in $\left[H_{\text {loc }}^{1}\left(\mathbb{R}, e^{c I d}\right)\right]^{N}$, we set $U^{ \pm}(x):=a^{ \pm}+\rho^{ \pm}(x) n^{ \pm}(x)$. Then $\left|\left(U^{ \pm}\right)_{x}\right|^{2}=\left(\left(\rho^{ \pm}\right)_{x}\right)^{2}+\left(\rho^{ \pm}\right)^{2}\left|n_{x}^{ \pm}\right|^{2}$. For any $I \subseteq \mathbb{R}$ measurable, we shall interpret integrals expressed in polar form as

$$
\int_{I}\left|U_{x}\right|^{2} e^{c x} d x=\int_{I \cap\left\{\rho^{ \pm}>0\right\}}\left\{\left(\rho_{x}^{ \pm}\right)^{2}+\left(\rho^{ \pm}\right)^{2}\left|n_{x}^{ \pm}\right|^{2}\right\} e^{c x} d x,
$$

since the imbedding $\left[H_{\text {loc }}^{1}(\mathbb{R})\right]^{N} \hookrightarrow\left[C_{\text {loc }}^{0}(\mathbb{R})\right]^{N}$ implies $\left|U_{x}\right|=0$ a.e. on the closed sets $\left\{U=a^{ \pm}\right\}$, even when they have positive measure. For any $\mu<\nu$ in $\mathbb{R}$, we set

$$
E_{c}(U,(\mu, \nu)):=\int_{\mu}^{\nu}\left\{\frac{1}{2}\left|U_{x}\right|^{2}+W(U)\right\} e^{c x} d x .
$$

This is the action (3) restricted on $[\mu, \nu]$.

Lemma 3 (cf. $\mathrm{A}-\mathrm{F}$ ). Assume $W$ satisfies (h) and $c>0$ is fixed. Let $a \in\left\{a^{+}, a^{-}\right\}$ and $U \in\left[H^{1}(\mu, \nu)\right]^{N}$ with $U=a+\rho n$, and suppose that

(i) $0<\rho(\mu)=\rho(\nu)=r \leq R_{0}\left(R_{0}\right.$ as in (h) $)$,

(ii) $r \leq \rho(x) \leq R_{0}$, for all $x \in(\mu, \nu)$.

Then, there exists a $\widetilde{U} \in\left[H^{1}(\mu, \nu)\right]^{N}, \widetilde{U}=a+\widetilde{\rho} n$, such that $U(\mu)=\widetilde{U}(\mu), U(\nu)=$ $\widetilde{U}(\nu)$ and $\widetilde{\rho}(x)<r$, for all $x \in(\mu, \nu)$ while

$$
E_{c}(\widetilde{U},(\mu, \nu))<E_{c}(U,(\mu, \nu))
$$

\footnotetext{
${ }^{1}$ We owe this argument to the referee.
} 
In particular, locally minimizing solutions to $U_{x x}-\nabla W(U)=-c U_{x}$ on $[\mu, \nu]$ attain the maximum value $r$ of their polar radius $\rho^{ \pm}=\left|U-a^{ \pm}\right|$only at the endpoints $\{\mu\},\{\nu\}$.

Proof of Lemma 3. We note that the proof of Lemma 3.3 in $\mathrm{A}-\mathrm{F}$ ] is based on a pointwise deformation and thus it holds generally for functionals of the form $\int\left(\frac{1}{2}\left|U_{x}\right|^{2}+W(U)\right) d \mu(x)$ with $\mu$ a positive Radon measure. See Lemma 10 for a similar argument.

We now prove that in view of (h), the polar radii of $U_{L}$ are weak subsolutions of the operator $L(\rho):=\rho_{x x}+c \rho_{x}$ in $\left[H^{1}(\mu, \nu)\right]^{N}$, for all $\mu<\nu<-L$ and $L<\mu<\nu$ (cf. Stefanopoulos [Stef]).

Proposition 4 (Constrained minimizers as radially weak $H^{1}$ subsolutions). The minimizers $U_{L}$ of Theorem 2 satisfy

$$
-\left(\rho_{L}^{ \pm}\right)_{x x}-c\left(\rho_{L}^{ \pm}\right)_{x}+\rho_{L}^{ \pm}\left|\left(n_{L}^{ \pm}\right)_{x}\right|^{2}+\nabla W\left(a^{ \pm}+\rho_{L}^{ \pm} n_{L}^{ \pm}\right) \cdot n_{L}^{ \pm} \leq 0,
$$

weakly in $H_{\mathrm{loc}}^{1}\left((L, \infty) \cap\left\{\rho_{L}^{+}>0\right\}\right)$ and $H_{\mathrm{loc}}^{1}\left((-\infty,-L) \cap\left\{\rho_{L}^{-}>0\right\}\right)$. In particular, if $W$ satisfies (h), we obtain

$$
\left(\rho_{L}^{ \pm}\right)_{x x}+c\left(\rho_{L}^{ \pm}\right)_{x} \geq 0
$$

Proof of Proposition 4. We construct local variations that do not violate the constraint $\rho_{L}^{ \pm} \leq r_{0}$. For definiteness we consider the case $a=a^{-}$; the other is similar. We take $\phi(x):=\theta(x) n_{L}^{-}(x)$ with $\theta$ in $C_{c}^{\infty}(-\infty,-L)$ and consider one-sided variations of the form

$$
U_{L}^{\varepsilon}(x):=U_{L}(x)-\varepsilon \phi(x)=a^{-}+\left(\rho_{L}^{-}(x)-\varepsilon \theta(x)\right) n_{L}^{-}(x)
$$

(radially inside the cylinder) which satisfy the constraint for small $\varepsilon \in\left[0, \varepsilon_{\phi}\right]$. Since $U_{L}$ is a minimizer of $E_{c}, E_{c}\left(U_{L}^{\varepsilon}\right) \geq E_{c}\left(U_{L}\right)$, for all $\varepsilon \in\left[0, \varepsilon_{\phi}\right]$. Consequently,

$$
\lim _{\varepsilon \rightarrow 0^{+}}\left[\frac{1}{\varepsilon}\left(E_{c}\left(U_{L}^{\varepsilon}\right)-E_{c}\left(U_{L}\right)\right)\right] \geq 0 \text {. }
$$

We calculate, using that $\operatorname{supp}(\theta) \subseteq(-\infty,-L)$,

$$
\begin{aligned}
E_{c}\left(U_{L}^{\varepsilon}\right)= & \int_{-\infty}^{-L}\left\{\frac{1}{2}\left(\left(\rho_{L}^{-}\right)_{x}-\varepsilon \theta_{x}\right)^{2}+\frac{1}{2}\left(\rho_{L}^{-}-\varepsilon \theta\right)^{2}\left|\left(n_{L}^{-}\right)_{x}\right|^{2}\right. \\
& \left.+W\left(a^{-}+\left(\rho_{L}^{-}-\varepsilon \theta\right) n_{L}^{-}\right)\right\} e^{c x} d x \\
& +\int_{-L}^{\infty}\left\{\frac{1}{2}\left|\left(U_{L}\right)_{x}\right|^{2}+W\left(U_{L}\right)\right\} e^{c x} d x .
\end{aligned}
$$

Taking one-sided $\left.\frac{d}{d \varepsilon}\right|_{\varepsilon=0^{+}}$, we get

$$
\int_{-\infty}^{-L}\left\{-\left(\rho_{L}^{-}\right)_{x}\left(\theta_{x} e^{c x}\right)-\left[\rho_{L}^{-}\left|\left(n_{L}^{-}\right)_{x}\right|^{2}+\nabla W\left(a^{-}+\rho_{L}^{-} n_{L}^{-}\right) \cdot n_{L}^{-}\right]\left(\theta e^{c x}\right)\right\} d x \geq 0 .
$$

We write $\theta_{x} e^{c x}=\left(\theta e^{c x}\right)_{x}-c \theta e^{c x}$ and substitute to get

$$
\int_{-\infty}^{-L}\left\{\left(\rho_{L}^{-}\right)_{x}\left(\theta e^{c x}\right)_{x}+\left[\rho_{L}^{-}\left|\left(n_{L}^{-}\right)_{x}\right|^{2}-\nabla W\left(a^{-}+\rho_{L}^{-} n_{L}^{-}\right) \cdot n_{L}^{-}\right]\left(\theta e^{c x}\right)\right\} d x \leq 0 .
$$


We are done, since the multiplication operator $M_{e^{\text {cId }}}$ is a Fréchet automorphism on the dense subspace $C_{c}^{\infty}(-\infty,-L)$ of $H_{\mathrm{loc}}^{1}(-\infty,-L)$.

It is now straightforward that all $\left(U_{L}\right)_{L \geq 1}$ realize the constraint at most at the rims of the cylinders.

Proposition 5 (Contact at most at the rims of the cylinders). If $W$ satisfies (h) , then

a) If $x_{L}^{+}:=\inf \left\{t \in \mathbb{R}: \rho_{L}^{+} \leq r_{0}\right.$ on $\left.[t,+\infty)\right\}$, then we have $\rho_{L}^{+}<r_{0}$ on $\left(x_{L}^{+},+\infty\right)$.

b) If $x_{L}^{-}:=\sup \left\{t \in \mathbb{R}: \rho_{L}^{-} \leq r_{0}\right.$ on $\left.(-\infty, t]\right\}$, then we have $\rho_{L}^{-}<r_{0}$ on $\left(-\infty, x_{L}^{-}\right)$.

Proof of Proposition 5. We drop sub/superscripts $L, \pm$ for $\rho$ and prove only a), since b) is analogous. By definition, $x_{L}^{+} \in(-L, L]$ and it is the time at which $U_{L}$ enters $\mathbb{B}\left(a^{+}, r_{0}\right)$ and remains inside it for all later times. Minimizers $U_{L}$ are, by (h), radially weak $H^{1}$ subsolutions: $\rho_{x x}+c \rho_{x} \geq 0$. Let $x_{0} \in\left(x_{L}^{+}, \infty\right)$ be such that $\rho\left(x_{0}\right)=r_{0}$. Since the point $x_{0}$ lies in the interior of $\left[x_{L}^{+}, x_{0}+1\right]$, by the Strong Maximum Principle for weak $C^{0}$ subsolutions ( $\left.\mathrm{G}-\mathrm{T}\right]$ ), we have that either $\rho\left(x_{0}\right)<r$, or $\rho \equiv r_{0}$ on $\left[x_{L}^{+}, x_{0}+1\right]$. Lemma 3 implies that $\rho$ is not identically $r_{0}$; otherwise we obtain a contradiction to minimality of $U_{L}$. Hence, $\rho<r_{0}$ on $\left(x_{L}^{+},+\infty\right)$.

Proposition 6 (Constrained minimizers are piecewise solutions). All $U_{L}$ are solutions to $U_{x x}-\nabla W(U)=-c U_{x}$ in $\left[C_{\mathrm{loc}}^{2}\left(\mathbb{R} \backslash\left\{x_{L}^{ \pm}\right\}\right)\right]^{N} \cap\left[C^{0}(\mathbb{R})\right]^{N}$. They are in $\left[C_{\mathrm{loc}}^{2}(\mathbb{R})\right]^{N}$ except possibly when $x_{L}^{ \pm}= \pm L$.

Proof of Proposition [6. By Proposition [5. $\left|U_{L}(x)-a^{ \pm}\right|<r_{0}$, for all $x \in \mathbb{R} \backslash\left[x_{L}^{-}, x_{L}^{+}\right]$. Take any point $x^{*} \in \mathbb{R} \backslash\left\{x_{L}^{-}, x_{L}^{+}\right\}$. By continuity, there exists an $\varepsilon_{0}>0$ and a compact tubular neighborhood $\left\{\mathbb{B}\left(U_{L}(x), \varepsilon_{0}\right): x \in\left[x^{*}-\delta, x^{*}+\delta\right]\right\}$ of the graph of $U_{L}$ not intersecting the boundary of the constraint cylinders, the assertion being trivial when $x^{*} \in\left(x_{L}^{-}, x_{L}^{+}\right)$. This holds for $x_{L}^{ \pm}$as well, when $x_{L}^{+}<L$ and $x_{L}^{-}>-L$. We take as variations of $U_{L}$ the $U_{L}^{\varepsilon}:=U_{L}-\varepsilon \phi,|\varepsilon| \leq \varepsilon_{0}$ small, for all $\phi$ in $\left[C_{c}^{\infty}\left(x^{*}-\delta-\varepsilon_{1}, x^{*}+\delta+\varepsilon_{1}\right)\right]^{N}, \varepsilon_{1}>0$ small, whose restriction on $\left(x^{*}-\delta, x^{*}+\delta\right)$ is dense in $\left[H^{1}\left(x^{*}-\delta, x^{*}+\delta\right)\right]^{N}$. Using that $\phi_{x} e^{c x}=\left(\phi e^{c x}\right)_{x}-c \phi e^{c x}$, we easily get that $U_{x x}-\nabla W(U)=-c U_{x}$ is solved weakly. Since $\nabla W \in\left[C_{\mathrm{loc}}^{1}\left(\mathbb{R}^{N}\right)\right]^{N}$ and $\left(U_{L}\right)_{x} \in\left[L_{\text {loc }}^{2}(\mathbb{R})\right]^{N}$, there exists $\left(U_{L}\right)_{x x} \in\left[L_{\text {loc }}^{2}\left(\mathbb{R} \backslash\left\{x_{L}^{ \pm}\right\}\right)\right]^{N}$ and therefore $U_{L} \in\left[C_{\text {loc }}^{1}\left(\mathbb{R} \backslash\left\{x_{L}^{ \pm}\right\}\right)\right]^{N}$ which gives that $U_{L} \in\left[C_{\text {loc }}^{2}\left(\mathbb{R} \backslash\left\{x_{L}^{ \pm}\right\}\right)\right]^{N}$, since $\nabla W \in$ $\left[C_{\text {loc }}^{1}\left(\mathbb{R}^{N}\right)\right]^{N}$.

Remark 7 (i). (Polar form of the equation). Write the equation $U_{x x}-\nabla W(U)=$ $-c U_{x}$ in polar coordinates $U_{L}=a^{ \pm}+\rho_{L}^{ \pm} n_{L}^{ \pm}$and multiply by $n_{L}^{ \pm}$to get that the polar radii $\rho_{L}^{ \pm}$of $U_{L}$ satisfy the equation

$$
(\rho)_{x x}+c(\rho)_{x}=\rho\left|n_{x}\right|^{2}+\nabla W\left(a^{ \pm}+\rho n\right) \cdot n .
$$

(ii) (Energy formula) Integrating the equation once as in the proof of Lemma 1 we get the formula

$$
c \int_{\mu}^{\nu}\left|U_{x}\right|^{2} d x=\left.\left(W(U)-\frac{\left|U_{x}\right|^{2}}{2}\right)\right|_{\mu} ^{\nu},
$$

on any interval $[\mu, \nu]$, on which $U$ solves $U_{x x}-\nabla W(U)=-c U_{x}$ classically. 
Proposition 8 (Asymptotic behavior of constrained minimizers). If $W$ satisfies (h), then $U_{L}(x) \longrightarrow a^{ \pm}$as $x \rightarrow \pm \infty$. Moreover, the polar radii $\rho_{L}^{ \pm}$of $U_{L}$ are eventually strictly monotone inside the cylinders and also $\left(U_{L}\right)_{x}( \pm \infty)=0$ at least up to subsequence.

Proof of Proposition 8. We treat both cases together, dropping the indices \pm on $\rho_{L}$.

Claim 1. The polar radii are eventually strictly monotone in the cylinders.

Indeed, by Lemma 3 and the action minimality of $U_{L}, \rho$ cannot be identically constant on any subinterval of $\left(-\infty, x_{L}^{-}\right)$or $\left(x_{L}^{+}, \infty\right)$. Hence, by continuity of $\rho$, the set of critical points $A:=\left\{\rho_{x}=0\right\}$ is discrete. Since $\rho$ solves $\rho_{x x}+c \rho_{x} \geq 0$, the Maximum Principle implies that $A$ does not contain maximum points. Moreover, $A$ cannot contain more than one minimum point; if a minimum point exists, then at all later points (in the unbounded direction of time) $\rho_{x}$ preserves its sign on both sides of the critical point. Hence, $\rho$ is eventually strictly monotone.

Now let $r^{*}$ denote the asymptotic limit of $\rho$. At $+\infty$ it readily follows that $r^{*}=0$, since $e^{c I d} W\left(U_{L}\right)$ is in $L^{1}(L, \infty)$. Indeed,

$$
\begin{aligned}
\int_{L}^{\infty} W^{+}\left(U_{L}\right) e^{c x} d x & \leq E_{c}\left(U_{\mathrm{aff}}\right)+\int_{-\infty}^{L} W^{-}\left(U_{L}\right) e^{c x} d x \\
& \leq E_{c}\left(U_{\mathrm{aff}}\right)+\frac{W^{-}\left(a^{-}\right) e^{c L}}{c}<\infty
\end{aligned}
$$

and $a^{+}$is the only zero of $W$ inside the ball $\mathbb{B}\left(a^{+}, r_{0}\right)$. Now we consider the limit at $-\infty$.

Claim 2. For any $t \in \mathbb{R}$ such that $[t, t+1] \subseteq\left(-\infty, x_{L}^{-}\right)$, we have

$$
0 \leq \min _{\substack{t \leq s \leq t+1 \\|\xi|=1}}\left[\nabla W\left(a^{-}+\rho(s) \xi\right) \cdot \xi\right] \leq \rho_{x}(t+1) e^{c}-\rho_{x}(t) .
$$

Indeed, since $U_{x x}-\nabla W(U)=-c U_{x}$ is solved by $U_{L}$ on $\left(-\infty, x_{L}^{-}\right)$, we integrate once the $e^{c x}$-multiple of equation (6) on $[t, t+1]$ to find

$$
\begin{aligned}
\int_{t}^{t+1}\left(\rho_{x} e^{c x}\right)_{x} d x & =\int_{t}^{t+1} e^{c x}\left(\nabla W\left(a^{-}+\rho n\right) \cdot n+\rho\left|n_{x}\right|^{2}\right) d x \\
& \geq e^{c t} \int_{t}^{t+1}\left(\nabla W\left(a^{-}+\rho n\right) \cdot n+\rho\left|n_{x}\right|^{2}\right) d x \\
& \geq e^{c t} \int_{t}^{t+1} \nabla W\left(a^{-}+\rho n\right) \cdot n d x \\
& \geq e^{c t} \min _{\substack{s \in[t, t+1]\\
}}\left[\nabla W\left(a^{-}+\rho(s) n(s)\right) \cdot n(s)\right] \\
& \geq e^{c t} \min _{\substack{t \leq \leq \leq t+1 \\
|\xi|=1}}\left[\nabla W\left(a^{-}+\rho(s) \xi\right) \cdot \xi\right] .
\end{aligned}
$$

Utilizing assumption (h), we obtain (8).

Since the limit of $\rho$ at $-\infty$ exists, there exists a sequence $x_{n} \longrightarrow-\infty$ such that $\rho_{x}\left(x_{n}\right) \longrightarrow 0$. Suppose first that eventually $\rho_{x} \geq 0$. By setting $t:=x_{n}-1$ in (8) 
and employing the monotonicity of $\rho$, we have

$$
0 \leq \min _{|\xi|=1}\left[\nabla W\left(a^{-}+\rho\left(x_{n}-1\right) \xi\right) \cdot \xi\right] \leq \rho_{x}\left(x_{n}\right) e^{c} .
$$

By employing that $\rho\left(x_{n}-1\right) \longrightarrow r^{*}$ and that $\rho_{x}\left(x_{n}\right) \longrightarrow 0$ as $n \longrightarrow \infty$, in the limit we obtain $\nabla W\left(a^{-}+r^{*} \xi\right) \cdot \xi=0$ for some $\xi$. Since $a^{-}$is the only critical point in $\mathbb{B}\left(a^{-}, r_{0}\right)$, it follows that $r^{*}=0$. Similarly, if $\rho_{x} \leq 0$, we take $t:=x_{n}$ to get

$$
0 \leq \min _{|\xi|=1}\left[\nabla W\left(a^{-}+\rho\left(x_{n}\right) \xi\right) \cdot \xi\right] \leq\left|\rho_{x}\left(x_{n}\right)\right|
$$

and again by passing to the limit as $n \longrightarrow \infty$ it follows that $r^{*}=0$.

Now we consider the convergence of the derivative. By multiplying (6) by $\rho$ and adding $\left(\rho_{x}\right)^{2}$, we obtain the identity

$$
\left|U_{x}\right|^{2}+\rho \nabla W(U) \cdot n=\frac{1}{2}\left[\left(\rho^{2}\right)_{x x}+c\left(\rho^{2}\right)_{x}\right] .
$$

Since $\rho^{2}$ is also strictly increasing and has a limit at $-\infty$, we get $\left(\rho^{2}\right)_{x} \geq 0$ and that there exists a sequence $\xi_{n} \longrightarrow-\infty$ such that $\left(\rho^{2}\right)_{x}\left(\xi_{n}\right) \longrightarrow 0$. By (9), assumption (h) and integration on $\left[\xi_{n}-1, \xi_{n}\right]$, we get

$$
\begin{aligned}
0 \leq \int_{\xi_{n}-1}^{\xi_{n}}\left|U_{x}\right|^{2} d x & \leq \frac{1}{2}\left[\left(\rho^{2}\right)_{x}\left(\xi_{n}\right)-\left(\rho^{2}\right)_{x}\left(\xi_{n}-1\right)\right]+\frac{c}{2}\left[\rho^{2}\left(\xi_{n}\right)-\rho^{2}\left(\xi_{n}-1\right)\right] \\
& \leq \frac{1}{2}\left[\left(\rho^{2}\right)_{x}\left(\xi_{n}\right)+c \rho^{2}\left(\xi_{n}\right)\right] \longrightarrow 0
\end{aligned}
$$

as $n \longrightarrow \infty$. The proof is complete.

We conclude this section by proving that $\left(U_{L}\right)_{x} \in\left[L^{2}(\mathbb{R})\right]^{N}$, but not necessarily $L$-uniformly. In addition, $U_{L}$ satisfies the first formula of Lemma 1 approximately, up to some additional terms which relate $c$ with the jump of $\left(U_{L}\right)_{x}$ at the rims.

Proposition 9 (Approximate relation for $c$ ). The 1-sided derivatives $\left(U_{L}\right)_{x}\left( \pm L^{ \pm}\right)$ of $U_{L}$ exist, and

$$
\begin{aligned}
c \int_{\mathbb{R}}\left|\left(U_{L}\right)_{x}\right|^{2} d x=W^{-}\left(a^{-}\right) & +\frac{1}{2}\left(\left|\left(U_{L}\right)_{x}\left(-L^{+}\right)\right|^{2}-\left|\left(U_{L}\right)_{x}\left(-L^{-}\right)\right|^{2}\right) \\
& +\frac{1}{2}\left(\left|\left(U_{L}\right)_{x}\left(+L^{+}\right)\right|^{2}-\left|\left(U_{L}\right)_{x}\left(+L^{-}\right)\right|^{2}\right) .
\end{aligned}
$$

In particular, $\left(U_{L}\right)_{x} \in\left[L^{2}(\mathbb{R})\right]^{N}$.

Proof of Proposition 9. Proposition [6 assures that we can apply formula (7) on $(-\infty,-L-\varepsilon),(-L+\varepsilon, L-\delta)$ and $(L+\delta, \infty)$ for $\varepsilon, \delta>0$ small utilizing by Proposition 8 the asymptotic behavior of the $U_{L}$ 's and the continuity of $W$. We obtain three relations on these intervals. Utilizing Hölder's inequality, we easily 
find that

$$
\begin{aligned}
\left|\left(U_{L}\right)_{x}(-L-\varepsilon)\right| \leq & \sqrt{2}\left(W\left(U_{L}(-L-\varepsilon)\right)+W^{-}\left(a^{-}\right)\right)^{\frac{1}{2}} \\
\left|\left(U_{L}\right)_{x}(-L+\varepsilon)\right| \leq & \sqrt{2}\left(c e^{c(L-\varepsilon)} \int_{-L+\varepsilon}^{L-\delta}\left|\left(U_{L}\right)_{x}\right|^{2} e^{c x} d x-W\left(U_{L}(+L-\delta)\right)\right. \\
& \left.+W\left(U_{L}(-L+\varepsilon)\right)+\frac{1}{2}\left|\left(U_{L}\right)_{x}(+L-\delta)\right|^{2}\right)^{\frac{1}{2}}, \\
\left|\left(U_{L}\right)_{x}(+L-\delta)\right| \leq & \sqrt{2}\left(W\left(U_{L}(L-\delta)\right)-W\left(U_{L}(-L+\varepsilon)\right)+\frac{1}{2}\left|\left(U_{L}\right)_{x}(-L+\varepsilon)\right|^{2}\right)^{\frac{1}{2}} \\
\left|\left(U_{L}\right)_{x}(+L+\delta)\right| \leq & \sqrt{2}\left(c e^{-c(L+\delta)} \int_{L+\delta}^{\infty}\left|\left(U_{L}\right)_{x}\right|^{2} e^{c x} d x+W\left(U_{L}(L+\delta)\right)\right)^{\frac{1}{2}} .
\end{aligned}
$$

Letting $\varepsilon \longrightarrow 0^{+}$and $\delta \longrightarrow 0^{+}$separately, we obtain that the moduli of the onesided limits exist, but may differ. Adding these relations and letting $\varepsilon, \delta \longrightarrow 0^{+}$ we obtain the formula for $c$.

\section{The RePlacement Lemmas}

We recall some basics from Differential Geometry. The canonical coordinates $(p, d)$ on $\mathbb{R}^{N}$ with respect to a $C^{2}$ convex set $\mathcal{C} \subseteq \mathbb{R}^{N}$ are defined by

$$
u=: p+d n,
$$

where $p$ is the projection on the convex set $\mathcal{C}, 0 \in \mathcal{C}, d$ the signed distance from $\partial \mathcal{C}$ and $n$ the outward unit normal of $\partial \mathcal{C}$. The latter is parameterized by the $C^{2}$ local coordinates

$$
\mathbb{R}^{N-1} \ni s=\left(s_{1}, \ldots, s_{N-1}\right) \mapsto p\left(s_{1}, \ldots, s_{N-1}\right) \in \partial \mathcal{C} .
$$

We may assume that the set of vectors

$$
\frac{\partial p}{\partial s_{i}}=\overrightarrow{t_{i}}, \quad i=1, \ldots, N-1,
$$

is an orthonormal frame in the tangent space at $p$, coinciding with the principal curvature directions ([DC], p. 144, p. 216). Thus,

$$
\frac{\partial n}{\partial s_{i}}=\kappa_{i} \overrightarrow{t_{i}}, \quad \kappa_{i}=\kappa_{i}(s) \text { the } i \text {-th principal curvature of } \partial \mathcal{C} .
$$

The coordinate system $(p, d)$ is defined for $-d_{0} \leq d$, provided that $d_{0} \kappa_{i} \leq 1$, $i=1, \ldots, N-1$ ( $[\mathrm{G}-\mathrm{T}]$ ). The orientation is such that $\kappa_{i} \geq 0$ when $\mathcal{C}$ is convex. We write

$$
U(x)=p(x)+d(x) n(x),
$$

meaning $p(x)=p(s(x)), n(x)=n(s(x))$. By differentiating (13),

$$
\begin{aligned}
\dot{U}(x) & =\dot{p}(x)+\dot{d}(x) n(x)+d(x) \dot{n}(x) \\
& =\vec{t}_{i} \dot{s}_{i}+\dot{d} n+d \kappa_{i} \vec{t}_{i} \dot{s}_{i} .
\end{aligned}
$$


Hence,

$$
|\dot{U}(x)|^{2}=\sum_{i=1}^{N-1} \dot{s}_{i}^{2}\left(1+\kappa_{i} d(x)\right)^{2}+(\dot{d}(x))^{2} .
$$

Now let $\mathcal{C}^{\prime} \subseteq \mathbb{R}^{N}$ be a convex set and assume that

$$
\nabla W \cdot n \geq \frac{c_{0}}{2}>0 \quad \text { on } \partial \mathcal{C}^{\prime}
$$

where $W \in C^{1}\left(\mathbb{R}^{N}\right)$ and $(p, d)$ are the canonical coordinates associated to $\partial \mathcal{C}^{\prime}$. By the $C^{1}$ smoothness of $W$ and (15), there is a $\bar{d}>0$ such that

$$
d \longmapsto W(p+d n) \quad \text { is increasing for }-\bar{d} \leq d \leq \bar{d} .
$$

Lemma 10. Let $x_{1}<x_{2}$ in $\mathbb{R}$ and $U \in\left[H^{1}\left(x_{1}, x_{2}\right)\right]^{N}$ be such that (i) $d\left(x_{1}\right)=d\left(x_{2}\right)=0$,

(ii) $0 \leq d(x) \leq \bar{d}$, for $x \in\left(x_{1}, x_{2}\right)$.

If (15) and (16) are satisfied, then there exists $\tilde{U} \in\left[H^{1}\left(x_{1}, x_{2}\right)\right]^{N}$ with the following properties:

$$
\begin{aligned}
\tilde{U}\left(x_{1}\right) & =U\left(x_{1}\right), \quad \tilde{U}\left(x_{2}\right)=U\left(x_{2}\right), \\
-\bar{d} & \leq \tilde{d}(x)<0, \text { for } x \in\left(x_{1}, x_{2}\right), \\
E_{\mu}\left(\tilde{U},\left(x_{1}, x_{2}\right)\right) & <E_{\mu}\left(U,\left(x_{1}, x_{2}\right)\right),
\end{aligned}
$$

where $\tilde{U}(x)=\tilde{p}(x)+\tilde{d}(x) n(x)$ and

$$
E_{\mu}\left(U,\left(x_{1}, x_{2}\right)\right):=\int_{x_{1}}^{x_{2}}\left(\frac{1}{2}|\dot{U}(x)|^{2}+W(U(x))\right) d \mu(x)
$$

where $\mu$ is any positive Radon measure on $\mathbb{R}$.

Proof of Lemma 10 (cf. Lemma 3.3 in $[\mathrm{A}-\mathrm{F}]$ ). Let $\phi:[0,1] \longrightarrow \mathbb{R}$ be a smooth function such that $\phi(0)=\phi(1)=0, \phi(\sigma)>0$ for $\sigma \in(0,1)$. For small $\varepsilon \geq 0$ define

$$
\widetilde{U}^{\varepsilon}(x):=p(x)-\varepsilon \phi\left(\frac{x-x_{1}}{x_{2}-x_{1}}\right) n(x), \quad x \in\left[x_{1}, x_{2}\right],
$$

where $U(x)=p(x)+d(x) n(x)$. By (14), we have

$$
|\dot{U}(x)|^{2}=\sum_{i=1}^{N-1} \dot{s}_{i}^{2}(x)+d^{2} \sum_{i=1}^{N-1} \kappa_{i}^{2} \dot{s}_{i}^{2}(x)+2 d \sum_{i=1}^{N-1} \kappa_{i} \dot{s}_{i}^{2}(x)+\dot{d}^{2}(x) .
$$

We note that

$$
\left|\dot{\tilde{U}}^{\varepsilon}\right|^{2}=\sum_{i=1}^{N-1} \dot{s}_{i}^{2}+\varepsilon^{2} \phi^{2} \sum_{i=1}^{N-1} \kappa_{i}^{2} \dot{s}_{i}^{2}-2 \varepsilon \phi \sum_{i=1}^{N-1} \kappa_{i} \dot{s}_{i}^{2}+\varepsilon^{2} \frac{\phi^{\prime 2}}{\left(x_{2}-x_{1}\right)^{2}} .
$$


Thus, we have that

$$
\begin{aligned}
E_{\mu}\left(\tilde{U}^{\varepsilon},\left(x_{1}, x_{2}\right)\right)= & E_{\mu}\left(\tilde{U}^{0},\left(x_{1}, x_{2}\right)\right) \\
& -\varepsilon \int_{x_{1}}^{x_{2}} \phi \sum_{i=1}^{N-1} \kappa_{i} \dot{s}_{i}^{2} d \mu+\frac{\varepsilon^{2}}{2} \int_{x_{1}}^{x_{2}} \phi^{2} \sum_{i=1}^{N-1} \kappa_{i}^{2} \dot{s}_{i}^{2} d \mu \\
& -\int_{x_{1}}^{x_{2}}(W(p)-W(p-\varepsilon \phi n)) d \mu \\
& +\frac{\varepsilon^{2}}{\left(x_{2}-x_{1}\right)^{2}} \int_{x_{1}}^{x_{2}} \phi^{\prime 2} d \mu .
\end{aligned}
$$

By (16), (ii) above and convexity of $\mathcal{C}^{\prime}$ we have

$$
E_{\mu}\left(\tilde{U}^{0},\left(x_{1}, x_{2}\right)\right) \leq E_{\mu}\left(U,\left(x_{1}, x_{2}\right)\right) .
$$

On the other hand, (16) also implies

$$
\begin{aligned}
-\int_{x_{1}}^{x_{2}}(W(p)- & W(p-\varepsilon \phi n)) d \mu+\frac{\varepsilon^{2}}{2\left(x_{2}-x_{1}\right)^{2}} \int_{x_{1}}^{x_{2}} \phi^{\prime 2} d \mu \\
= & -\int_{x_{1}}^{x_{2}}\left(\int_{0}^{1} \frac{d}{d \tau}(W(p-\varepsilon \tau \phi n)) d \tau\right) d \mu \\
& +\frac{\varepsilon^{2}}{2\left(x_{2}-x_{1}\right)^{2}} \int_{x_{1}}^{x_{2}} \phi^{\prime 2} d \mu \\
= & -\varepsilon \int_{x_{1}}^{x_{2}}\left(\int_{0}^{1} W_{u}(p-\varepsilon \tau \phi n) \cdot \phi n\right) d \tau d \mu \\
& +\frac{\varepsilon^{2}}{2\left(x_{2}-x_{1}\right)^{2}} \int_{x_{1}}^{x_{2}} \phi^{\prime 2} d \mu \\
\frac{155}{<} & -C \varepsilon+\frac{\varepsilon^{2}}{2\left(x_{2}-x_{1}\right)^{2}} \int_{x_{1}}^{x_{2}} \phi^{\prime 2} d \mu<0
\end{aligned}
$$

for some $C>0$ and small $\varepsilon>0$. Finally, we observe that by the convexity of $\mathcal{C}^{\prime}$,

$$
-\varepsilon \int_{x_{1}}^{x_{2}} \phi \sum_{i=1}^{N-1} \kappa_{i} \dot{s}_{i}^{2} d \mu+\frac{\varepsilon^{2}}{2} \int_{x_{1}}^{x_{2}} \phi \sum_{i=1}^{N-1} \kappa_{i}^{2} \dot{s}_{i}^{2} d \mu \leq 0,
$$

for small $\varepsilon>0$. From these inequalities and (17), the lemma follows with $\tilde{U}:=\tilde{U}^{\varepsilon}$, $0<\varepsilon \ll 1$.

Hypotheses. (H1) $W: \mathbb{R}^{N} \longrightarrow \mathbb{R}, C^{2}$, with two minima $W\left(a^{-}\right)<W\left(a^{+}\right)=0$. $(\mathrm{H} 2)\{u \mid W(u) \leq 0\}=: \mathcal{C}_{0}^{-} \cup\left\{a^{+}\right\}, \mathcal{C}_{0}^{-}$compact, convex.

(H3) (i) $\nabla W \cdot n \geq c_{0}>0$ on $\partial \mathcal{C}_{0}^{-}=:\{W=0\}_{(-)}, n$ the outward unit normal on $\partial \mathcal{C}_{0}^{-}$.

(ii) $\nabla^{2} W \geq c_{0} I$ on $\{W=0\}_{(-)}$.

Remark 11. a) By $C^{2}$ smoothness of $W$, there exists a $b>0$ such that

$$
\nabla^{2} W \leq b I, \quad \text { on }\{u \mid W(u) \leq 0\} .
$$


b) (H3) implies that the set $\{u \mid W(u)=\beta\}$ for $0<\beta \ll 1$ is made up of two components, which we denote by

$$
\{W=\beta\}_{(-)} \quad \text { and } \quad\{W=\beta\}_{(+)},
$$

with $\{W=\beta\}_{(-)}$convex and enclosing $a^{-}$. On the other hand, for $\beta<0(|\beta| \ll 1)$, $\{u \mid W(u)=\beta\}$ is made up of one component which is convex. So more precisely there is an $\alpha_{0}>0$ such that $\{W=\beta\}_{(-)}$is convex, $-\alpha_{0} \leq \beta \leq \alpha_{0}$. By the smoothness of $W$,

$$
W_{u} \cdot n \geq \frac{c_{0}}{2} \quad \text { on }\{W=\beta\}_{(-)},-\alpha_{0} \leq \beta \leq \alpha_{0} .
$$

Note that the sets $\{W=\beta\}_{(-)}$are nested for $-\alpha_{0} \leq \beta \leq \alpha_{0}$.

Now we take $\alpha \in\left(0, \alpha_{0}\right)$ and furthermore restrict it as follows:

$$
0<\alpha<\frac{c_{0}}{4} \lambda=: \bar{a}_{0},
$$

where $\lambda$ is a fixed number satisfying the conditions

$$
0 \leq \lambda \leq \frac{c_{0}}{2 b}, \quad 0<\lambda \leq d_{0}, \quad \lambda<\frac{1}{\max \left\{\kappa_{1}, \ldots, \kappa_{N-1}\right\}},
$$

with $b$ as in (20) above,

$$
d_{0}=\operatorname{dist}\left(\left\{W=\alpha_{0}\right\}_{(-)},\left\{W=-\alpha_{0}\right\}_{(-)}\right),
$$

and $\kappa_{1}, \ldots, \kappa_{N-1}$ the principal curvatures of $\{W=\beta\}_{(-)}$(all positive by convexity). We note that

$$
W(p-\lambda n(p))<0, \quad \text { for } p \in\{W=\alpha\}_{(-)} .
$$

Indeed (dropping $p$ in $n(p)$ ),

$$
\begin{aligned}
W(p)-W(p-\lambda n) & =-\int_{0}^{\lambda} \frac{d}{d t}[W(p-t n)] d t \\
& =\int_{0}^{\lambda}(\nabla W(p-t n)-\nabla W(p)+\nabla W(p)) \cdot n d t \\
& =\int_{0}^{\lambda} \nabla W(p) \cdot n d t-\int_{0}^{\lambda} \int_{t}^{0} \frac{d}{d s}(\nabla W(p-s n) d s) \cdot n d t \\
& =\int_{0}^{\lambda} \nabla W(p) \cdot n d t-\int_{0}^{\lambda} \int_{0}^{t} \nabla^{2} W(p-s n) n \cdot n d s d t \\
& \geq \frac{c_{0}}{2} \lambda-\frac{b}{2} \lambda^{2} \quad(\underline{(20)},(\underline{21})) \\
& \geq \frac{c_{0}}{4} \lambda \quad \quad\left(\lambda \leq \frac{c_{0}}{2 b}\right) .
\end{aligned}
$$

Therefore, we have

$$
W(p)-\frac{c_{0}}{4} \lambda \geq W(p-\lambda n)
$$

and so (by (22) )

$$
0>\alpha-\frac{c_{0}}{4} \lambda \geq W(p-\lambda n) .
$$


Lemma 12. Let $\mathcal{C}$ denote the component of $\{u \mid W(u) \geq \alpha\}$ with $\partial \mathcal{C}=\{W=\alpha\}_{(-)}$. Let $(p, d)$ be the canonical coordinates with respect to $\mathcal{C}$. Assume that $\alpha$ is as in (22), and assume that (H1), (H2), and (H3) hold. Also let $x_{1}<x_{2} \in \mathbb{R}$ and $U \in\left[H^{1}\left(x_{1}, x_{2}\right)\right]^{N}$ be such that

(i) $d\left(x_{1}\right)=d\left(x_{2}\right)=0$,

(ii) $d\left(x_{0}\right) \geq 0$, for some $x_{0} \in\left(x_{1}, x_{2}\right)$.

Then, there is a $\tilde{U} \in\left[H^{1}\left(x_{1}, x_{2}\right)\right]^{N}$ with the properties

$$
\tilde{U}\left(x_{1}\right)=U\left(x_{1}\right), \quad \tilde{U}\left(x_{2}\right)=U\left(x_{2}\right),
$$

where $-d_{0} \leq \tilde{d}(x)<0$, for $x \in\left(x_{1}, x_{2}\right)$, and

$$
E_{\mu}\left(\tilde{U},\left(x_{1}, x_{2}\right)\right)<E_{\mu}\left(U,\left(x_{1}, x_{2}\right)\right),
$$

where $\tilde{U}(x)=\tilde{p}(x)+\tilde{d}(x) n(x)$.

Proof of Lemma 12 (cf. Lemma 3.4 in $[\mathrm{A}-\mathrm{F}]$ ). Let

$$
\rho_{M}:=\max _{x \in\left[x_{1}, x_{2}\right]} d(x) .
$$

We can assume that $d\left(x_{0}\right)=\rho_{M}$. We first analyze the case $d\left(x_{0}\right)=\rho_{M}=0$. In this case we can assume that $d(x)<0$ for some $x \in\left(x_{1}, x_{0}\right)\left(x \in\left(x_{0}, x_{2}\right)\right)$, since otherwise, by Lemma 10, we can replace $U$ with a function that satisfies this condition and has less action. From this and the continuity of $U$, the existence of $\hat{x}_{1} \in\left(x_{1}, x_{0}\right), \hat{x}_{2} \in\left(x_{0}, x_{2}\right),-\frac{d_{0}}{2}<\hat{d}<0$, such that $d\left(\hat{x}_{1}\right)=d\left(\hat{x}_{2}\right)=\hat{d}$ and $\hat{d}<d(x)<0$, for $x \in\left(\hat{x}_{1}, \hat{x}_{2}\right)$ follows. We now consider the parallel hypersurface to $\partial \mathcal{C}$, parameterized by $p+\hat{d} n(p), p \in \partial \mathcal{C}$. This is convex, and we denote it by $\partial \mathcal{C}^{\prime}$. It can be deduced by (21) that condition (15) holds on $\partial \mathcal{C}^{\prime}$. Then we can apply Lemma 10 on $\partial \mathcal{C}^{\prime}$ and obtain a local replacement between $\hat{x}_{1}$ and $\hat{x}_{2}$ and conclude that the claim of the lemma is true if $\rho_{M}=0$. Therefore we can assume that $\rho_{M}>0$. If $0<\rho_{M} \leq d_{0}$, again we can conclude the proof by using Lemma 10 applied to the connected component $I_{0}$ of the set $\left\{x \in\left(x_{1}, x_{2}\right) \mid d(x)>0\right\}$ that contains $x_{0}$. It remains to analyze the case $\rho_{M}>d_{0}$. We can identify $\left(x_{1}, x_{2}\right)$ with $I_{0}$. Let $h:\left[0, d_{0}\right] \longrightarrow[-\lambda, 0], h(\sigma)=-\lambda \frac{\sigma}{d_{0}}$. Then $h(0)=0, h\left(d_{0}\right)=-\lambda$. We define the deformation

$$
\tilde{U}(x):= \begin{cases}p(x)+h(d(x)) n(x), & \text { for } x \in\left[x_{1}, x_{2}\right], d(x)<d_{0}, \\ p(x)-\lambda n(x), & \text { for } x \in\left[x_{1}, x_{2}\right], d(x) \geq d_{0}\end{cases}
$$

$\tilde{U}\left(x_{1}\right)=U\left(x_{1}\right), \tilde{U}\left(x_{2}\right)=U\left(x_{2}\right)$. For the kinetic energy we have the estimates

$$
\begin{aligned}
|\dot{U}(x)|^{2} & =\sum_{i=1}^{N-1} \dot{s}_{i}^{2}\left(1+\kappa_{i} d(x)\right)^{2}+\dot{d}^{2}(x) \\
& \geq \sum_{i=1}^{N-1} \dot{s}_{i}^{2}\left(1+\kappa_{i} h\right)^{2}+\left(h^{\prime}(d)\right)^{2} \dot{d}^{2}(x) \\
& =|\dot{\tilde{U}}(x)|^{2}
\end{aligned}
$$


when $d(x)<d_{0}$, while for $d(x) \geq d_{0}$ we have

$$
\begin{aligned}
|\dot{U}(x)|^{2} & =\sum_{i=1}^{N-1} \dot{s}_{i}^{2}\left(1+\kappa_{i} d(x)\right)^{2}+\dot{d}^{2}(x) \\
& \geq \sum_{i=1}^{N-1} \dot{s}_{i}^{2}\left(1+\kappa_{i} d(x)\right)^{2} \\
& >\sum_{i=1}^{N-1} \dot{s}_{i}^{2}\left(1-\lambda \kappa_{i}\right)^{2} \\
& =|\dot{\tilde{U}}(x)|^{2} .
\end{aligned}
$$

Hence,

$$
\int_{x_{1}}^{x_{2}}|\dot{\tilde{U}}(x)|^{2} d \mu(x)<\int_{x_{1}}^{x_{2}}|\dot{U}(x)|^{2} d \mu(x) .
$$

For the potential energy we have the estimates

$$
\begin{aligned}
W(\tilde{U}(x)) & =W(p(x)+h(d(x)) n(x)) \\
& =W\left(p(x)-\frac{\lambda d(x)}{d_{0}} n(x)\right) \\
& \leq W(p(x)+d(x) n(x)) \quad(\text { by (21) }) \\
& =W(U(x)),
\end{aligned}
$$

when $d(x)<d_{0}$, while for $d(x) \geq d_{0}$ we have by (23) and (H2) that

$$
W(\tilde{U}(x)) \leq 0 \leq W(U(x)) .
$$

Putting it all together, we have

$$
\int_{x_{1}}^{x_{2}} W(\tilde{U}(x)) d \mu(x)<\int_{x_{1}}^{x_{2}} W(U(x)) d \mu(x) .
$$

The argument so far establishes that

$$
E_{\mu}\left(\tilde{U},\left(x_{1}, x_{2}\right)\right)<E_{\mu}\left(U,\left(x_{1}, x_{2}\right)\right) .
$$

The proof of Lemma 12 is complete.

\section{ACTION PROPERTIES OF MINIMIZERS}

We now show that $E_{c}\left(U_{L}\right)$ is a function of the jumps at the rims $\left|\left(U_{L}\right)_{x}\left( \pm L^{+}\right)\right|^{2}-$ $\left|\left(U_{L}\right)_{x}\left( \pm L^{-}\right)\right|^{2}$, while $E_{c}\left(U_{L}\right)=0$ for minimizers in $\left[C^{2}(\mathbb{R})\right]^{N}$ which solve $U_{x x}-$ $\nabla W(U)=-c U_{x}$ on $\mathbb{R}$. To prove this, we derive an equipartition relation at $+\infty$ (see $\mathrm{A}-\mathrm{Be}-\mathrm{C}], \mathrm{A}-\mathrm{F}$ ] and our result, Lemma 15). We first need a formula for the action of solutions:

Lemma 13 (1st integral). Every solution to $U_{x x}-\nabla W(U)=-c U_{x}$ in $\left[C^{2}(\mu, \nu)\right]^{N}$ satisfies:

$$
E_{c}(U,(\mu, \nu))=\int_{\mu}^{\nu}\left\{\frac{1}{2}\left|U_{x}\right|^{2}+W(U)\right\} e^{c x} d x=\left.\left\{\frac{e^{c x}}{c}\left(W(U)-\frac{\left|U_{x}\right|^{2}}{2}\right)\right\}\right|_{\mu} ^{\nu} .
$$


Proof of Lemma 13. The equation $U_{x x}-\nabla W(U)=-c U_{x}$ implies $-U_{x x} \cdot U_{x}+$ $\nabla W(U) \cdot U_{x}=c\left|U_{x}\right|^{2}$; hence we obtain

$$
\left(\frac{1}{2}\left|U_{x}\right|^{2}-W(U)\right)_{x}=-c\left|U_{x}\right|^{2}
$$

Integrating by parts the $e^{c x}$-multiple of this equation, we get

$$
\begin{aligned}
\left.\left\{\frac{e^{c x}}{2}\left|U_{x}\right|^{2}\right\}\right|_{\mu} ^{\nu} & -\frac{c}{2} \int_{\mu}^{\nu}\left|U_{x}\right|^{2} e^{c x} d x-\left.\left(e^{c x} W(U)\right)\right|_{\mu} ^{\nu}+c \int_{\mu}^{\nu} W(U) e^{c x} d x \\
& =-c \int_{\mu}^{\nu}\left|U_{x}\right|^{2} e^{c x} d x,
\end{aligned}
$$

which leads to the desired formula.

Lemma 14 (The action in terms of the jumps). The minimizers $U_{L}$ satisfy

$$
\begin{aligned}
E_{c}\left(U_{L}\right)= & \lim _{\omega \rightarrow \infty} \frac{e^{c \omega}}{c}\left(W\left(U_{L}(\omega)\right)-\frac{\left|\left(U_{L}\right)_{x}(\omega)\right|^{2}}{2}\right) \\
& +\frac{e^{+c L}}{2 c}\left(\left|\left(U_{L}\right)_{x}\left(+L^{+}\right)\right|^{2}-\left|\left(U_{L}\right)_{x}\left(+L^{-}\right)\right|^{2}\right) \\
& \left.+\frac{e^{-c L}}{2 c}\left(\left|\left(U_{L}\right)_{x}\left(-L^{+}\right)\right|^{2}-\left|\left(U_{L}\right)_{x}\left(-L^{-}\right)\right|^{2}\right)\right\}=: e_{c}\left(U_{L}\right) .
\end{aligned}
$$

The quantity $e_{c}\left(U_{L}\right)$ comprises "error terms" which vanish if $U_{L} \in\left[C_{\mathrm{loc}}^{2}(\mathbb{R})\right]^{N}$.

Proof of Lemma 14. First note that $E_{c}\left(U_{L}\right)=\lim _{\omega \rightarrow \infty} E_{c}\left(U_{L},(-\infty, \omega)\right)$. Apply Lemma 13 to $U_{L}$ which is a piecewise solution on $(-\infty,-L),(-L, L),(L, \omega)$ and add the three relations, utilizing the continuity of $W\left(U_{L}\right)$ at $\pm L$. Finally, let $\omega \longrightarrow \infty$.

Solutions to $U_{x x}=\nabla W(U)$ in the well-studied case of $c=0$ satisfy an equipartition property: $2 W(U)=\left|U_{x}\right|^{2}$. Our dissipation term $-c\left|U_{x}\right|^{2}$ forces a similar behavior but at $+\infty$.

Lemma 15 (Asymptotic equipartition of the energy at $+\infty$ ). The minimizers $U_{L}$ satisfy

$$
\lim _{\omega \rightarrow \infty}\left[\frac{e^{c \omega}}{c}\left(W\left(U_{L}(\omega)\right)-\frac{\left|\left(U_{L}\right)_{x}(\omega)\right|^{2}}{2}\right)\right]=0 .
$$

Proof of Lemma 15. By the formula (7) for $\mu=\omega, \nu=\infty$ and Proposition 8, we have

This gives

$$
0 \leq c \int_{\omega}^{\infty}\left|\left(U_{L}\right)_{x}\right|^{2} d x=\frac{\left|\left(U_{L}\right)_{x}(\omega)\right|^{2}}{2}-W\left(U_{L}(\omega)\right) .
$$

$$
\begin{aligned}
0 \leq \frac{e^{c \omega}}{c}\left(\frac{\left|\left(U_{L}\right)_{x}(\omega)\right|^{2}}{2}-W\left(U_{L}(\omega)\right)\right) & =e^{c \omega} \int_{\omega}^{\infty}\left|\left(U_{L}\right)_{x}\right|^{2} d x \\
& \leq \int_{\omega}^{\infty}\left|\left(U_{L}\right)_{x}\right|^{2} e^{c x} d x .
\end{aligned}
$$

By Proposition 9, we have $\left(U_{L}\right)_{x} \in\left[L^{2}\left(\mathbb{R}, e^{c I d}\right)\right]^{N}$. Hence, letting $\omega \longrightarrow \infty$ we are done. 
Corollary 16 (The action measures the jump discontinuities). We have that $E_{c}\left(U_{L}\right)=e_{c}\left(U_{L}\right)$, with $e_{c}\left(U_{L}\right)$ as in Lemma 14. In particular, $E_{c}\left(U_{L}\right)=0$ if $U_{L} \in\left[C_{l o c}^{2}(\mathbb{R})\right]^{N}$.

\section{ImPlications of THE LOCAL REPLACEMENT LEMMAS. DETERMINATION OF THE SPEED}

We first introduce our main hypothesis on the potential (cf. (H1)-(H3) in Section 4):

$W$ is in $C_{\text {loc }}^{2}\left(\mathbb{R}^{N}\right), a^{ \pm}$are minima, $W\left(a^{-}\right)<0=W\left(a^{+}\right)$and $\min _{\mathbb{R}^{N}}\{W\}$ $=W\left(a^{-}\right)$. Moreover:

(1) There is an $\alpha_{0}>0$ such that for all $\alpha \in\left(0, \alpha_{0}\right]$, we have $W^{-1}(\{\alpha\})=$ $\partial \mathcal{C}_{\alpha}^{-} \cup \partial \mathcal{C}_{\alpha}^{+},\left\{u \in \mathbb{R}^{N} \mid W \leq \alpha\right\}=\mathcal{C}_{\alpha}^{-} \cup \mathcal{C}_{\alpha}^{+}$, where $\mathcal{C}_{\alpha}^{-}, \mathcal{C}_{\alpha}^{+}$are disjoint compact, convex sets with $C^{2}$ boundaries, containing $a^{ \pm}$, respectively. Moreover, $\nabla W \cdot n \geq c_{0}>0$ on $\partial \mathcal{C}_{0}^{-}$and $\nabla^{2} W \geq c_{0} I$ on $\partial \mathcal{C}_{0}^{-}, n$ the outward unit normal of $\partial \mathcal{C}_{0}^{-}$.

(2) The map $r \mapsto W\left(a^{-}+r \xi\right)$ has a strictly positive derivative as long as $a^{-}+r \xi \in \mathcal{C}_{\alpha}^{-},|\xi|=1, r>0$.

Assumption (h*) implies that $\liminf _{|u| \rightarrow \infty}\{W(u)\} \geq \alpha_{0}$. Thus $W$ satisfies

$$
W^{-1}\left(\left[W\left(a^{-}\right), 0\right]\right) \subset \subset \mathbb{R}^{N},
$$

which was assumed in Theorem 2 .

Definition 17. For $\alpha \in\left(0, \bar{\alpha}_{0}\right]$ and $L \geq 1$, we set

$$
\begin{aligned}
\lambda_{L}^{-} & :=\sup \left\{x \in \mathbb{R}:\left|U_{L}(x)-a^{-}\right|=r_{0}\right\}, \\
\lambda_{L}^{+} & :=\inf \left\{x \in \mathbb{R}:\left|U_{L}(x)-a^{+}\right|=r_{0}\right\}, \\
\lambda_{L}^{\alpha-}:=\sup \{x \in \mathbb{R}: & \left.U_{L}(x) \in \partial\left(\mathcal{C}_{\alpha}^{-}\right)\right\} .
\end{aligned}
$$

We will show that $U_{L}$ intersects exactly once any of the sets $\partial \mathbb{B}\left(a^{-}, r_{0}\right), \partial \mathbb{B}\left(a^{+}, r_{0}\right)$, $\partial \mathcal{C}_{\alpha}^{-}$. Decreasing $\alpha>0$ if necessary, we may assume $\mathcal{C}_{a}^{+} \subseteq \mathbb{B}\left(a^{+}, r_{0}\right)$ and that $\mathbb{B}\left(a^{+}, r_{0}\right)$ is disjoint from $\mathcal{C}_{a}^{-}$.

Proposition 18 (Global a priori control on action minimizers). Assume $W$ satisfies (h) and (므, $\alpha$ is as in Definition 17 and let $\left(U_{L}\right)_{L \geq 1}$ be the family of minimizers of Theorem 2, For all $L \geq 1$, we have

(I) $U_{L}$ exits $\mathcal{C}_{\alpha}^{-}$precisely once at $x=\lambda_{L}^{\alpha-}$, that is,

$$
x \in\left(-\infty, \lambda_{L}^{\alpha-}\right] \quad \Longrightarrow W\left(U_{L}(x)\right) \leq \alpha .
$$

(II) The image $U_{L}(\mathbb{R})$ restricted to $\mathbb{R}^{N} \backslash\left(\mathcal{C}_{\alpha}^{-} \cup \mathbb{B}\left(a^{+}, r_{0}\right)\right)$ has only one connected component and

$$
W\left(U_{L}(x)\right) \geq \alpha \text { for } x \in\left[\lambda_{L}^{\alpha-}, \lambda_{L}^{+}\right]
$$

(III) The image $U_{L}(\mathbb{R})$ restricted on $\mathcal{C}_{\alpha}^{-} \cup \mathbb{B}\left(a^{+}, r_{0}\right)$ has precisely two connected components and

$$
W\left(U_{L}(x)\right) \leq \alpha \text { for some } x \in \mathbb{R} ; \text { then either } x \in\left(-\infty, \lambda_{L}^{\alpha}\right] \text { or } x \in\left[\lambda_{L}^{+},+\infty\right) \text {. }
$$


(IV) The numbers $\lambda_{L}^{ \pm}$are well defined as the unique times at which $U_{L}$ crosses the spheres $\partial\left(\mathbb{B}\left(a^{ \pm}, r_{0}\right)\right)$.

(V) The polar radii $\rho_{L}^{ \pm}=\left|U_{L}-a^{ \pm}\right|$are strictly monotone on $\left[\lambda_{L}^{+},+\infty\right),\left(-\infty, \lambda_{L}^{\alpha-}\right]$, respectively.

Proof of Proposition 18, 1. We first settle $\lambda_{L}^{-}$. We note that Lemma 3.4 of $[\mathrm{A}-\mathrm{F}$ ] applies because the local replacements in its proof are pointwise, and because $W\left(a^{-}\right) \leq W\left(a^{+}\right)$. Thus, $\lambda_{L}^{-}$is unique and half of (IV) is established.

2. Next we settle $\lambda_{L}^{\alpha-}$. By applying Lemma 12, we obtain the existence of a unique intersection of $U_{L}$ with $\partial \mathcal{C}_{a}^{-}$, and so (I) is established.

3 . We handle $\lambda_{L}^{+}$as follows. Assume by contradiction that $U_{L}$ intersects $\partial \mathbb{B}\left(a^{+}, r_{0}\right)$ more than once. Then, there are $x_{1}<x_{2}$ such that $U_{L}\left(x_{i}\right) \in \partial \mathbb{B}\left(a^{+}, r_{0}\right), i=1,2$ and $U_{L}\left(x_{i}\right) \notin \mathbb{B}\left(a^{+}, r_{0}\right), x_{1}<x<x_{2}$. Since by step 2 above, $U_{L}$ cannot intersect $\partial \mathcal{C}_{\alpha}^{-}$for those $x$ 's, it follows that Lemma 3.4 in $\mathrm{A}-\mathrm{F}$. applies and leads to a local replacement with less action and thus to a contradiction. Thus, by step 1 above, (IV) has been established.

4. The previous arguments show that $U_{L}(x)$ cannot exit $\mathcal{C}_{\alpha}^{-}$before $x=\lambda_{L}^{\alpha-}$ and cannot enter $\mathbb{B}\left(a^{+}, r_{0}\right)$ before $x=\lambda_{L}^{+}$. Thus we have control on the intervals for which $U_{L}$ is in the monotonicity regions, which implies the $L^{\infty}$ bounds

$$
\left\|\rho_{L}^{-}\right\|_{L^{\infty}\left(-\infty, \lambda_{L}^{\alpha-}\right)} \leq \max _{u \in \mathcal{C}_{\alpha}^{-}}\left|u-a^{-}\right|, \quad\left\|\rho_{L}^{+}\right\|_{L^{\infty}\left(\lambda_{L}^{+}, \infty\right)} \leq r_{0} .
$$

It follows that Lemma 3 can be applied to the minimizers inside $\mathbb{B}\left(a^{ \pm}, r\right)$ with $r>r_{0}$ showing that they cannot be identically constant on any subinterval. By (ㄱ), $\rho_{L}^{ \pm}$satisfy $\left(\rho_{L}^{ \pm}\right)_{x x}+c\left(\rho_{L}^{ \pm}\right)_{x} \geq 0$. By the Strong Maximum Principle, both $\rho_{L}^{ \pm}$ cannot have local maxima; thus they are strictly monotone. By Proposition 8 it follows that the same is true for $r<r_{0}$; thus (V) has been established.

Remark 19. We have the ordering $-L \leq \lambda_{L}^{\alpha-} \leq \lambda_{L}^{+}$. We will prove existence by showing that for some $L<\infty$ large, the constraint is not realized: $-L<\lambda_{L}^{-}$and $\lambda_{L}^{+}<L$ strictly. We define

$$
\lambda_{L}^{0-}:=\sup \left\{x \in \mathbb{R}: U_{L}(x) \in \partial \mathcal{C}_{0}^{-}\right\} .
$$

Note that $-L \leq \lambda_{L}^{0-} \leq \lambda_{L}^{\alpha-} \leq \lambda_{L}^{+}$.

In the sequel we will need the following estimate.

Lemma 20. If $\operatorname{dist}\left(\mathcal{C}_{\alpha}^{-}, \mathbb{B}\left(a^{+}, r_{0}\right)\right)=: d_{\alpha}$, then for all $\alpha \in\left[0, \bar{\alpha}_{0}\right]$ and $L \geq 1$, we have

$$
E_{c}\left(U_{L}\right) \geq-\frac{W^{-}\left(a^{-}\right)}{c} e^{c \lambda_{L}^{0-}}+\frac{\alpha}{c}\left[e^{c \lambda_{L}^{+}}-e^{c \lambda_{L}^{\alpha-}}\right]+\frac{c d_{\alpha}^{2}}{2\left(e^{-c \lambda_{L}^{\alpha-}}-e^{-c \lambda_{L}^{+}}\right)} .
$$

Proof of Lemma 20. We have the identity

$$
E_{c}\left(U_{L}\right)=-\int_{-\infty}^{\lambda_{L}^{0-}} W^{-}\left(U_{L}\right) e^{c x} d x+\int_{\lambda_{L}^{0-}}^{\infty} W^{+}\left(U_{L}\right) e^{c x} d x+\frac{1}{2} \int_{\mathbb{R}}\left|\left(U_{L}\right)_{x}\right|^{2} e^{c x} d x .
$$


We estimate each term separately, recalling that $W\left(U_{L}\right) \geq \alpha$ on $\left[\lambda_{L}^{\alpha-}, \lambda_{L}^{+}\right]$and $W^{-}\left(U_{L}\right) \leq W^{-}\left(a^{-}\right):$

$$
\begin{aligned}
\int_{-\infty}^{\lambda_{L}^{0-}} W^{-}\left(U_{L}\right) e^{c x} d x & \leq W^{-}\left(a^{-}\right) \int_{-\infty}^{\lambda_{L}^{0-}} e^{c x} d x \\
& =\frac{W^{-}\left(a^{-}\right)}{c} e^{c \lambda_{L}^{0-}}, \\
\int_{\lambda_{L}^{0-}}^{\infty} W^{+}\left(U_{L}\right) e^{c x} d x & \geq \int_{\lambda_{L}^{\alpha-}}^{\lambda_{L}^{+}} W^{+}\left(U_{L}\right) e^{c x} d x \\
& \geq \alpha \int_{\lambda_{L}^{\alpha-}}^{\lambda_{L}^{+}} e^{c x} d x=\frac{\alpha}{c}\left[e^{c \lambda_{L}^{+}}-e^{c \lambda_{L}^{\alpha-}}\right], \\
d_{\alpha} \leq\left|U_{L}\left(\lambda_{L}^{\alpha-}\right)-U_{L}\left(\lambda_{L}^{+}\right)\right| & \leq \int_{\lambda_{L}^{\alpha-}}^{\lambda_{L}^{+}}\left|\left(U_{L}\right)_{x}\right| d x \\
& \leq\left(\int_{\lambda_{L}^{\alpha-}}^{\lambda_{L}^{+}} e^{-c x} d x\right)^{\frac{1}{2}}\left(\int_{\lambda_{L}^{\alpha-}}^{\lambda_{L}^{+}}\left|\left(U_{L}\right)_{x}\right|^{2} e^{c x} d x\right)^{\frac{1}{2}} .
\end{aligned}
$$

Hence, we have

$$
d_{\alpha}^{2} \leq\left(\frac{e^{-c \lambda_{L}^{\alpha-}}-e^{-c \lambda_{L}^{+}}}{c}\right) \int_{\mathbb{R}}\left|\left(U_{L}\right)_{x}\right|^{2} e^{c x} d x .
$$

Putting these bounds together, we obtain the desired estimate.

The speed of the travelling wave. Thus far, all the results were valid for an arbitrary $c>0$. It is easy to see that the specific $c=c^{*}$ that guarantees existence should be very special: by Proposition 9 ,

$$
\begin{aligned}
\left(\left|\left(U_{L}\right)_{x}\left(+L^{+}\right)\right|^{2}-\left|\left(U_{L}\right)_{x}\left(+L^{-}\right)\right|^{2}\right) & +\left(\left|\left(U_{L}\right)_{x}\left(-L^{+}\right)\right|^{2}-\left|\left(U_{L}\right)_{x}\left(-L^{-}\right)\right|^{2}\right) \\
+2 W^{-}\left(a^{-}\right) & =2 c \int_{\mathbb{R}}\left|\left(U_{L}\right)_{x}\right|^{2} d x \\
& \geq 2 c \int_{-L}^{L}\left|\left(U_{L}\right)_{x}\right|^{2} d x \\
& \geq \frac{c\left|U_{L}(+L)-U_{L}(-L)\right|^{2}}{L} \\
& \geq \frac{c}{L}\left(\left|a^{+}-a^{-}\right|-2 r_{0}\right)^{2},
\end{aligned}
$$

which shows that if $c \longrightarrow+\infty$ we cannot achieve the smooth matching of piecewise solutions at any $L<\infty$. On the other hand, by Corollary [16] and the a priori bound (5), we have

$$
\begin{aligned}
e^{+c L}\left(\left|\left(U_{L}\right)_{x}\left(+L^{+}\right)\right|^{2}-\left|\left(U_{L}\right)_{x}\left(+L^{-}\right)\right|^{2}\right) & +e^{-c L}\left(\left|\left(U_{L}\right)_{x}\left(-L^{+}\right)\right|^{2}-\left|\left(U_{L}\right)_{x}\left(-L^{-}\right)\right|^{2}\right) \\
& =2 c E_{c}\left(U_{L}\right) \\
& \leq 2 c E_{c}\left(U_{\mathrm{aff}}\right) \\
& \leq-2 e^{-c} W^{-}\left(a^{-}\right)+2 c e^{c}\left(E_{0}^{+}\left(U_{\mathrm{aff}}\right)\right),
\end{aligned}
$$


which shows that derivatives cannot match if $c \longrightarrow 0^{+}$. The desired $c=c^{*}$ is the specific value, at which, for sufficiently large $L>L^{*} \geq 1, E_{c}\left(U_{L}\right)=0$. This behavior of $E_{c>0}$ is not present in its $E_{c=0}$ counterpart ([A-F], A-Be-C]), but it is plausible: $U_{x x}-\nabla W(U)=-c U_{x}$ is translation invariant while (3) is not. Translates $U(\cdot-\delta), \delta \neq 0$ of solutions occur as minimizers to a rescaled $e^{c \delta} E_{c}$, but both waves have the same action only if $E_{c}(U(\cdot-\delta))=E_{c}(U)=0$.

Remark 21. Note that for fixed $c>0$, the function $L \longmapsto E_{c}\left(U_{L}\right):[1, \infty) \longrightarrow$ $\left(-\infty, E_{c}\left(U_{\text {aff }}\right)\right]$ is nonincreasing in $L$ : as $L$ increases, $\mathcal{X}_{L}$ increases $\left(L<L^{\prime}\right.$ implies $\left.\mathcal{X}_{L} \subset \mathcal{X}_{L^{\prime}}\right)$ and $E_{c}\left(U_{L}\right)$ decreases (see Section 2 for definitions).

The next two estimates are key ingredients and will allow us to determine the speed and establish existence. The full strength of (h*) is employed to show that $U_{L}$ cannot get trapped for infinite time inside $\mathcal{C}_{\alpha}^{-}$, after exiting the ball $\mathbb{B}\left(a^{-}, r_{0}\right)$. We set

$$
R_{\max }^{\alpha}:=\max _{u \in \partial \mathcal{C}_{\alpha}^{-}}\left|u-a^{-}\right|
$$

Lemma 22. If $W$ satisfies (高), there exists a $w^{*}>0$ such that if $\alpha \in\left[0, \bar{\alpha}_{0}\right]$,

$$
\begin{aligned}
\lambda_{L}^{\alpha-}-\lambda_{L}^{-} & \leq \frac{1}{w^{*}}\left\{c R_{\max }^{\alpha}+\left[\left(c R_{\max }^{\alpha}\right)^{2}+2 w^{*}\left|R_{\max }^{\alpha}-r_{0}\right|\right]^{\frac{1}{2}}\right\} \\
& =: \Lambda_{\alpha,-} .
\end{aligned}
$$

As $w^{*}$ we may take

$$
w^{*}:=\min _{\substack{r_{0} \leq r \leq R_{\max }^{\alpha} \\|\xi|=1}}\left[\left.\frac{d}{d t}\right|_{t=r} W\left(a^{-}+t \xi\right)\right] .
$$

Proof of Lemma 22. Writing $U_{x x}-\nabla W(U)=-c U_{x}$ in the polar form $U_{L}=$

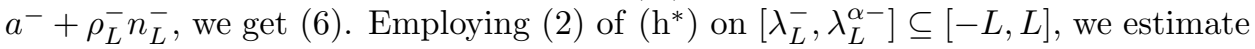

$$
\begin{aligned}
\left(\rho_{L}^{-}\right)_{x x}+c\left(\rho_{L}^{-}\right)_{x} & \geq \nabla W\left(a^{-}+\rho_{L}^{-} n\right) \cdot n_{L}^{-} \\
& =\left.\frac{d}{d t}\right|_{t=\rho_{L}^{-}} W\left(a^{-}+t n_{L}^{-}\right) \\
& \geq \min _{\substack{r_{0} \leq r \leq R_{\max }^{\alpha} \\
|\xi|=1}}\left[\left.\frac{d}{d t}\right|_{t=r} W\left(a^{-}+t \xi\right)\right]=: w^{*}>0 .
\end{aligned}
$$

Integrating once $\left(\rho_{L}^{-}\right)_{x x}+c\left(\rho_{L}^{-}\right)_{x} \geq w^{*}$ on $\left[\lambda_{L}^{-}, x\right], x \leq \lambda_{L}^{\alpha-}$ we get

$$
\left(\rho_{L}^{-}\right)_{x}+c \rho_{L}^{-} \geq w^{*}\left(x-\lambda_{L}^{-}\right)+\left\{c \rho_{L}^{-}\left(\lambda_{L}^{-}\right)+\left(\rho_{L}^{-}\right)_{x}\left(\left(\lambda_{L}^{-}\right)^{+}\right)\right\} .
$$

By Proposition [18, we have $\{\} \geq$.0 . By a further integration,

$$
\int_{\lambda_{L}^{-}}^{x}\left(\rho_{L}^{-}\right)_{z}(z) d z+c \int_{\lambda_{L}^{-}}^{x}\left(\rho_{L}^{-}\right)(z) d z \geq w^{*} \int_{\lambda_{L}^{-}}^{x}\left(z-\lambda_{L}^{-}\right) d z .
$$

Set $x:=\lambda_{L}^{\alpha-}$. We utilize the a priori bound $\left\|\rho_{L}^{-}\right\|_{L^{\infty}\left[\lambda_{L}^{-}, \lambda_{L}^{\alpha-}\right]} \leq R_{\max }^{\alpha}$ and the fact that the right term equals $\frac{w^{*}}{2}\left[\lambda_{L}^{\alpha-}-\lambda_{L}^{-}\right]^{2}$ to obtain

$$
\frac{\left|R_{\max }^{\alpha}-r_{0}\right|}{\lambda_{L}^{\alpha-}-\lambda_{L}^{-}}+\frac{c}{\lambda_{L}^{\alpha-}-\lambda_{L}^{-}}\left(\int_{\lambda_{L}^{-}}^{x}\left(\rho_{L}^{-}\right)(z) d z\right) \geq \frac{w^{*}}{2}\left[\lambda_{L}^{\alpha-}-\lambda_{L}^{-}\right],
$$


which gives the desired inequality. Setting $\lambda_{L}^{\alpha-}-\lambda_{L}^{-}=: x$ and comparing with the solutions of the parabola $\frac{w^{*}}{2} x^{2}-\left(c R_{\max }^{\alpha}\right) x-\left|R_{\max }^{\alpha}-r_{0}\right| \leq 0$ we obtain

$$
\frac{w^{*}}{2}\left[\lambda_{L}^{\alpha-}-\lambda_{L}^{-}\right] \leq \frac{\left|R_{\max }^{\alpha}-r_{0}\right|}{\lambda_{L}^{\alpha-}-\lambda_{L}^{-}}+c R_{\max }^{\alpha},
$$

which clearly implies (24).

Lemma 23. For all $\alpha \in\left(0, \bar{\alpha}_{0}\right]$, we have the implication:

$$
E_{c}\left(U_{L}\right) \leq 0 \Longrightarrow \lambda_{L}^{+}-\lambda_{L}^{\alpha-} \leq \frac{1}{c} \ln \left(1+\frac{W^{-}\left(a^{-}\right)}{\alpha}\right)=: \Lambda_{\alpha,+} .
$$

Proof of Lemma 23. This follows directly from the estimate of Lemma 20.

$$
\begin{aligned}
0 & \geq E_{c}\left(U_{L}\right) \\
& \geq e^{c \lambda_{L}^{\alpha-}}\left\{-\frac{W^{-}\left(a^{-}\right)}{c}+\frac{\alpha}{c}\left(e^{c\left(\lambda_{L}^{+}-\lambda_{L}^{\alpha-}\right)}-1\right)+\frac{c d_{\alpha}^{2}}{2\left(1-e^{-c\left(\lambda_{L}^{+}-\lambda_{L}^{\alpha-}\right)}\right)}\right\} \\
& \geq \frac{e^{c \lambda_{L}^{\alpha-}} \alpha}{c}\left\{-\left(\frac{W^{-}\left(a^{-}\right)}{\alpha}+1\right)+e^{c\left(\lambda_{L}^{+}-\lambda_{L}^{\alpha-}\right)}\right\} .
\end{aligned}
$$

Corollary 24. The length of the time interval $\left[\lambda_{L}^{-}, \lambda_{L}^{+}\right]$for which the graph of $U_{L}$ remains between the constraint cylinders is bounded uniformly in $L$ as long as $E_{c}\left(U_{L}\right) \leq 0$.

Proof of Corollary 24, By Lemmas 22 and 23, we have

$$
\begin{aligned}
\lambda_{L}^{+}-\lambda_{L}^{-} & =\left(\lambda_{L}^{+}-\lambda_{L}^{\alpha-}\right)+\left(\lambda_{L}^{\alpha-}-\lambda_{L}^{-}\right) \\
& \leq \Lambda_{\alpha,+}+\Lambda_{\alpha,-} \\
& =: \Lambda<\infty,
\end{aligned}
$$

provided that $E_{c}\left(U_{L}\right) \leq 0$. This proves the bound.

Proposition 25 (Determination of the speed of the travelling wave). There exist $c^{*}>0$ and $L^{*} \geq 1$ such that, for all $L \geq L^{*}$,

$$
E_{c^{*}}\left(U_{L}\right)=\inf _{\mathcal{X}_{L}}\left[E_{c^{*}}\right]=0 .
$$

The proof consists of several lemmas.

Lemma 26. For any $L \geq 1$ and any $V \in \mathcal{X}_{L}$, both fixed, the function $c \longmapsto E_{c}(V)$ is continuous on $F:=\left\{c>0:\left|E_{c}(V)\right|<\infty\right\}$.

Proof of Lemma 26. Let $c_{m} \longrightarrow c_{\infty}>0$ as $m \longrightarrow \infty$. Since $V \in \mathcal{X}_{L}$, we have $W(V)=W^{+}(V) \geq 0$ on $[L, \infty)$ and as a result, for any $c \in F$,

$$
\begin{aligned}
0 & \leq \int_{L}^{\infty}\left(\frac{1}{2}\left|V_{x}\right|^{2}+W(V)\right) e^{c x} d x \\
& =E_{c}(V)-\int_{-\infty}^{L}\left(\frac{1}{2}\left|V_{x}\right|^{2}+W(V)\right) e^{c x} d x \\
& \leq E_{c}(V)+\sup _{(-\infty, L]}|W(V)| \int_{-\infty}^{L} e^{c x} d x \\
& <\infty .
\end{aligned}
$$


Hence, for $m$ large we have on $(L,+\infty)$ that

$$
\left|\left(\frac{1}{2}\left|V_{x}\right|^{2}+W(V)\right) e^{c_{m} \mathrm{Id}}\right| \leq 2\left(\frac{1}{2}\left|V_{x}\right|^{2}+W(V)\right) e^{c_{\infty} \mathrm{Id}} \in L^{1}(L,+\infty) .
$$

Again for any $c \in F$, we have

$$
\begin{aligned}
\left.\int_{-\infty}^{L}\left|\frac{1}{2}\right| V_{x}\right|^{2}+W(V) \mid e^{c x} d x & \leq \int_{-\infty}^{L}\left(\left\{\frac{1}{2}\left|V_{x}\right|^{2}+W(V)\right\}+2|W(V)|\right) e^{c x} d x \\
& \leq E_{c}(V)+2 \sup _{(-\infty, L]}|W(V)| \int_{-\infty}^{L} e^{c x} d x \\
& <\infty
\end{aligned}
$$

Since $c_{m} \longrightarrow c_{\infty}$ as $m \longrightarrow \infty$, if we choose $m$ large enough such that $c_{m} \leq \frac{3}{2} c_{\infty}$, we have $e^{c_{m} x} \leq e^{c_{\infty} L} e^{\frac{c_{\infty}}{2} x}$ for all $x \leq L$. Hence, for $m$ large we have on $(-\infty, L)$ that

$$
\left|\left(\frac{1}{2}\left|V_{x}\right|^{2}+W(V)\right) e^{c_{m} \mathrm{Id}}\right| \leq e^{c_{\infty} L}\left(\frac{1}{2}\left|V_{x}\right|^{2}+W(V)\right) e^{\frac{c_{\infty}}{2} \mathrm{Id}} \in L^{1}(-\infty, L) .
$$

By the pointwise convergence $\left(\frac{1}{2}\left|V_{x}\right|^{2}+W(V)\right) e^{c_{m} \mathrm{Id}} \longrightarrow\left(\frac{1}{2}\left|V_{x}\right|^{2}+W(V)\right) e^{c_{\infty} \mathrm{Id}}$ as $m \longrightarrow \infty$, the lemma follows by application of the Dominated Convergence Theorem on $(-\infty, L)$ and $(L,+\infty)$ separately.

Recall that $U_{L}$ has so far always denoted the minimizer of $E_{c}$ into $\mathcal{X}_{L}$ for fixed $c$. We will temporarily denote the dependence of $U_{L}$ on $c$ explicitly by $U_{L, c}$. Following an idea of Heinze [Hei], we introduce the following set:

$$
C:=\left\{c>0 \mid \exists L \geq 1: E_{c}\left(U_{L, c}\right)<0\right\}
$$

Lemma 27. The set (27) is open, nonempty and $\sup C \leq \sqrt{2 W^{-}\left(a^{-}\right)} d_{0}{ }^{-1}$.

Proof of Lemma 27. By observing that $C$ equals the set

$$
\left\{c>0 \mid \exists L \geq 1 \& \exists V \in \mathcal{X}_{L}: E_{c}(V)<0\right\}
$$

Lemma 26 implies that $C$ is open. By the bound (5) on $U_{\text {aff }} \in \bigcap_{L \geq 1} \mathcal{X}_{L}$, we have $f(c) \geq E_{c}\left(U_{\text {aff }}\right)$, where

$$
f(c):=e^{-c}\left(-\frac{1}{c} W^{-}\left(a^{-}\right)+e^{2 c} E_{0}^{+}\left(U_{\mathrm{aff}}\right)\right) .
$$

Moreover, the equation $f(c)=0$ has a unique solution $c_{0}>0$ since $f$ changes sign and $f^{\prime}>0$ on $(0, \infty)$. Hence, $\left(0, c_{0}\right) \subseteq C \neq \emptyset$. Moreover, by Lemma 20, for $c \in C$ fixed, we have

$$
0>E_{c}(V) \geq E_{c}\left(U_{L}\right) \geq e^{c \lambda_{L}^{\alpha-}}\left[-\frac{W^{-}\left(a^{-}\right)}{c}+\frac{c d_{\alpha}^{2}}{2\left(1-e^{-c\left(\lambda_{L}^{+}-\lambda_{L}^{\alpha-}\right)}\right)}\right],
$$

which implies that $0 \geq c^{2} d_{\alpha}^{2}-2 W^{-}\left(a^{-}\right)$. Letting $\alpha \longrightarrow 0^{+}$, we finally obtain

$$
0<c_{0} \leq \sup C \leq \sqrt{2 W^{-}\left(a^{-}\right)} d_{0}^{-1}
$$

Lemma 28. Suppose that $L \geq 1$ is fixed and we have a sequence $C \ni c_{m} \longrightarrow c_{\infty}$ as $m \longrightarrow \infty, c_{\infty}>0$. Then, there exists a subsequence $c_{m, k} \longrightarrow c_{\infty}$ along which

$$
E_{c_{m, k}}\left(U_{L, c_{m, k}}\right) \longrightarrow E_{c_{\infty}}\left(U_{L, c_{\infty}}\right), \text { as } k \longrightarrow \infty \text {. }
$$


Proof of Lemma 28. Fix $\varepsilon>0$ and choose $V \in \mathcal{X}_{L}$ such that $E_{c_{\infty}}(V)-\varepsilon \leq$ $E_{c_{\infty}}\left(U_{L, c_{\infty}}\right) \leq E_{c_{\infty}}(V)$. Since $c_{m} \longrightarrow c_{\infty}$, by Lemma 26, we can choose $m(\varepsilon) \in N$ large such that $\left|E_{c_{\infty}}(V)-E_{c_{m}}(V)\right| \leq \varepsilon$, for all $m \geq m(\varepsilon)$. Thus,

$$
\begin{aligned}
E_{c_{m}}\left(U_{L, c_{m}}\right) & \leq E_{c_{m}}(V) \\
& \leq E_{c_{\infty}}(V)+\varepsilon \\
& \leq E_{c_{\infty}}\left(U_{L, c_{\infty}}\right)+2 \varepsilon,
\end{aligned}
$$

which implies

$$
\limsup _{m \rightarrow \infty} E_{c_{m}}\left(U_{L, c_{m}}\right) \leq E_{c_{\infty}}\left(U_{L, c_{\infty}}\right) .
$$

By arguing as in the proof of Theorem [2, there exists a subsequence $c_{m, k} \longrightarrow c_{\infty}$ along which $U_{L, c_{m, k}} \longrightarrow \bar{U}$ in $\left[C_{\mathrm{loc}}^{0}(\mathbb{R})\right]^{N}$ and $U_{L, c_{m}, k} \longrightarrow \bar{U}$ weakly in $\left[H_{\mathrm{loc}}^{1}(\mathbb{R})\right]^{N}$, as $k \longrightarrow \infty$. By weak LSC of the $L^{2}$ norm, we have

$$
\liminf _{k \rightarrow \infty} \frac{1}{2} \int_{\mathbb{R}}\left|\left(U_{L, c_{m, k}}\right)_{x}\right|^{2} e^{c_{m, k} x} d x \geq \frac{1}{2} \int_{\mathbb{R}}\left|\left(U_{L, c_{\infty}}\right)_{x}\right|^{2} e^{c_{\infty} x} d x
$$

For $k$ large, we have the lower bound

$$
W\left(U_{L, c_{m, k}}\right) e^{c_{m, k} \mathrm{Id}} \geq-\left(e^{c_{\infty} L} W^{-}\left(a^{-}\right)\right) e^{\frac{c_{\infty}}{2} \mathrm{Id}} \chi_{(-\infty, L]},
$$

which is an $L^{1}(\mathbb{R})$ function. Hence, the Fatou lemma implies

$$
\liminf _{k \rightarrow \infty} \int_{\mathbb{R}} W\left(U_{L, c_{m, k}}\right) e^{c_{m, k} x} d x \geq \int_{\mathbb{R}} W\left(U_{L, c_{\infty}}\right) e^{c_{\infty} x} d x .
$$

We conclude that

$$
\liminf _{k \rightarrow \infty} E_{c_{m, k}}\left(U_{L, c_{m, k}}\right) \geq E_{c_{\infty}}(\bar{U}) \geq E_{c_{\infty}}\left(U_{L, c_{\infty}}\right) .
$$

Putting (28) and (29) together, the proof follows.

Lemma 29. If $c^{*}:=\sup C$, then $E_{c^{*}}\left(U_{L, c^{*}}\right)=0$ for all $L \geq \Lambda$.

Proof of Lemma 29. By (27), there exists a sequence $C \ni c_{m} \longrightarrow c^{*}$ as $m \longrightarrow \infty$ such that $E_{c_{m}}\left(U_{L_{m}, c_{m}}\right)<0$. By the negativity of the action we may employ the bound (26) to obtain

$$
\lambda_{L_{m}}^{+}-\lambda_{L_{m}}^{-} \leq \Lambda,
$$

which is uniform in $m \in \mathbb{N}$. Moreover, since $E_{c_{m}}\left(U_{L_{m}, c_{m}}\right)<0$, we necessarily have $\lambda_{L_{m}}^{+}=L_{m}$, since otherwise a translation to the right would contradict the minimality of $U_{L_{m}, c_{m}}$. By observing that the translate $U_{L_{m}, c_{m}}\left(\cdot+L_{m}\right)$ is in $\mathcal{X}_{\Lambda}$, we have

$$
\begin{aligned}
E_{c_{m}}\left(U_{\Lambda, c_{m}}\right) & \leq E_{c_{m}}\left(U_{L_{m}, c_{m}}\left(\cdot+L_{m}\right)\right) \\
& =e^{-c_{m} L_{m}} E_{c_{m}}\left(U_{L_{m}, c_{m}}\right) \\
& <0 .
\end{aligned}
$$

By Lemma 28, the passage to the limit as $m \longrightarrow \infty$ (along a subsequence if necessary) implies

$$
\begin{aligned}
E_{c^{*}}\left(U_{\Lambda, c^{*}}\right) & =\lim _{m \rightarrow \infty} E_{c_{m}}\left(U_{\Lambda, c_{m}}\right) \\
& \leq 0 .
\end{aligned}
$$

Since $c^{*}=\sup C$ and $C$ is open, $c^{*} \notin C$ and as a result $E_{c^{*}}\left(U_{\Lambda, c^{*}}\right) \geq 0$. By Remark 21 and (27), we conclude that $E_{c^{*}}\left(U_{L, c^{*}}\right)=0$ for all $L \geq \Lambda$. 
Proof of Proposition 25. By putting Lemmas 26, 27, 28 and 29 together, the proof of Proposition 25 follows with $c^{*}=\sup C, L^{*}=\Lambda$.

Proposition 25 provides a $c^{*}$ for which $E_{c^{*}}\left(U_{L}\right)=0$ for large $L$ and this is sufficient for existence. However, $c^{*}$ is the unique possible speed of minimizing travelling waves 2

Proposition 30 (Uniqueness of the speed). Assume that a minimizing solution $(U, c)$ to (11) exists. Then, there exists precisely one constant $c_{*}$ such that $\left(U, c_{*}\right)$ solves (1).

Corollary 31. Since minimizers of (3) have vanishing action, we have $c_{*}=c^{*}$. Hence, Proposition 25 provides the unique constant for which $E_{c^{*}}(U)=0$.

Proof of Proposition 130, Let $\left(U_{1}, c_{1}^{*}\right),\left(U_{2}, c_{2}^{*}\right)$ be two solutions of (1) with $0<c_{1}^{*}<$ $c_{2}^{*}$ and possibly $U_{1}=U_{2}$. The differential form of the formula in Lemma 13 is

$$
\frac{\left|U_{x}\right|^{2}}{2}+W(U)=e^{-c x}\left(\frac{e^{c x}}{c}\left[W(U)-\frac{\left|U_{x}\right|^{2}}{2}\right]\right)_{x} .
$$

We set $c:=c_{2}^{*}, U:=U_{2}^{*}$, multiply by $e^{c_{1}^{*} x}$ and integrate by parts the right hand side to obtain

$$
\begin{aligned}
\int_{-t}^{t} e^{c_{1}^{*} x}\left(\frac{\left|\left(U_{2}\right)_{x}\right|^{2}}{2}+W\left(U_{2}\right)\right) d x= & \left.\left(\frac{e^{c_{1}^{*} x}}{c_{2}^{*}}\left[W\left(U_{2}\right)-\frac{\left|\left(U_{2}^{*}\right)_{x}\right|^{2}}{2}\right]\right)\right|_{-t} ^{t} \\
& -\left(c_{1}^{*}-c_{2}^{*}\right) \int_{-t}^{t} \frac{e^{c_{1}^{*} x}}{c_{2}^{*}}\left[W\left(U_{2}\right)-\frac{\left|\left(U_{2}\right)_{x}\right|^{2}}{2}\right] d x
\end{aligned}
$$

We rewrite this identity as

$$
\begin{aligned}
\left.\left(e^{c_{1}^{*} x}\left[W\left(U_{2}\right)-\frac{\left|\left(U_{2}^{*}\right)_{x}\right|^{2}}{2}\right]\right)\right|_{-t} ^{t}= & c_{2}^{*} \int_{-t}^{t} e^{c_{1}^{*} x}\left(\frac{\left|\left(U_{2}\right)_{x}\right|^{2}}{2}+W\left(U_{2}\right)\right) d x \\
& +c_{1}^{*} \int_{-t}^{t} e^{c_{1}^{*} x}\left[W\left(U_{2}\right)-\frac{\left|\left(U_{2}\right)_{x}\right|^{2}}{2}\right] d x \\
& -c_{2}^{*} \int_{-t}^{t} e^{c_{1}^{*} x}\left[W\left(U_{2}\right)-\frac{\left|\left(U_{2}\right)_{x}\right|^{2}}{2}\right] d x \\
= & c_{2}^{*} \int_{-t}^{t} e^{c_{1}^{*} x}\left|\left(U_{2}\right)_{x}\right|^{2} d x \\
& -c_{1}^{*} \int_{-t}^{t} e^{c_{1}^{*} x} \frac{\left|\left(U_{2}\right)_{x}\right|^{2}}{2} d x \\
& +c_{1}^{*} \int_{-t}^{t} e^{c_{1}^{*} x} W\left(U_{2}\right) d x \\
= & \left(c_{2}^{*}-c_{1}^{*}\right) \int_{-t}^{t} e^{c_{1}^{*} x}\left|\left(U_{2}\right)_{x}\right|^{2} d x \\
& +c_{1}^{*} E_{c_{1}^{*}}\left(U_{2},(-t, t)\right) .
\end{aligned}
$$

Hence, we have the identity

$$
c_{1}^{*} E_{c_{1}^{*}}\left(U_{2},(-t, t)\right)=\left(c_{1}^{*}-c_{2}^{*}\right) \int_{-t}^{t}\left|\left(U_{2}\right)_{x}\right|^{2} e^{c_{1}^{*} x} d x+\left.\left(e^{c_{1}^{*} x}\left[W\left(U_{2}\right)-\frac{\left|\left(U_{2}\right)_{x}\right|^{2}}{2}\right]\right)\right|_{-t} ^{t} .
$$

\footnotetext{
${ }^{2}$ This fact together with a sketch of its proof has been kindly pointed out by the referee.
} 
By Proposition (8), $\left(U_{2}\right)_{x} \longrightarrow 0$ as $t \rightarrow \pm \infty$ up to subsequences. Since $E_{c_{2}^{*}}\left(U_{2}\right)=0$, we have

$$
\begin{aligned}
\int_{\mathbb{R}}\left\{\frac{\left|\left(U_{2}\right)_{x}\right|^{2}}{2}+W^{+}\left(U_{2}\right)\right\} e^{c_{2}^{*} x} d x & =\int_{\mathbb{R}} W^{-}\left(U_{2}\right) e^{c_{2}^{*} x} d x \\
& \leq W^{-}\left(a^{-}\right) \frac{e^{c_{2}^{*} L_{2}^{*}}}{c_{2}^{*}} \\
& <\infty
\end{aligned}
$$

where $L_{2}^{*}$ is a large constant as in Proposition 25. Hence, since $c_{1}^{*}<c_{2}^{*}$ we may let $t \rightarrow \infty$ to obtain

$$
c_{1}^{*} E_{c_{1}^{*}}\left(U_{2}\right)=\left(c_{1}^{*}-c_{2}^{*}\right) \int_{\mathbb{R}}\left|\left(U_{2}\right)_{x}\right|^{2} e^{c_{1}^{*} x} d x<0 .
$$

But this contradicts that $c_{1}^{*} E_{c_{1}^{*}}\left(U_{2}\right) \geq 0$.

We therefore assume in the remaining that $c=c^{*}$, the unique speed provided by Proposition 25.

A variational characterization of minimizing travelling waves. Summarizing, solutions $(U, c)$ to the system of equations

$$
E_{c}(U)=\inf \left\{E_{c}(V): V \in\left[H_{\mathrm{loc}}^{1}(\mathbb{R})\right]^{N}, V( \pm \infty)=a^{ \pm}\right\}, \quad E_{c}(U)=0,
$$

are heteroclinic travelling waves and solve the differential equations

$$
\left\{\begin{array}{l}
U_{x x}-\nabla W(U)=-c U_{x} \\
U( \pm \infty)=a^{ \pm}
\end{array}\right.
$$

Both the weight $e^{c I d}$ of (3) and its minimizer are unknown. The first equation of the system involves the minimization problem for $E_{c}$ in the class $\left\{E_{c} \mid c>0\right\}$, and the second one selects $c=c^{*}$ so that the minimum is zero.

\section{Removing the COnstraints}

In this section we prove the existence of a solution to problem (11).

Theorem 32 (Existence). Assume that the potential $W$ satisfies (h), (h*). Then, there exists a travelling wave solution $(U, c) \in\left[C^{2}(\mathbb{R})\right]^{N} \times(0,+\infty)$ to

$$
\left\{\begin{array}{l}
U_{x x}-\nabla W(U)=-c U_{x} \\
U( \pm \infty)=a^{ \pm}
\end{array}\right.
$$

The speed $c$ equals the constant $c^{*}$ in Proposition 25, which is unique. In particular, $E_{c^{*}}(U)=0$.

Proof of Theorem 32. By Proposition 25, we have $E_{c^{*}}\left(U_{L, c^{*}}\right)=0$, for all $L \geq L^{*}$. By Corollary 24, if we choose $L>\Lambda$ we obtain a minimizer $U:=U_{L}$ of $E_{c}$ with $c=c^{*}$ for which $E_{c}(U)=0$. Thus either $U$ or a translate $U(\cdot-\delta)$ (with necessarily the same action) does not realize the constraint, solving (1) on $\mathbb{R}$. The proof is complete. 
Corollary 33. The speed $c^{*}$ has the variational characterization ${ }^{3}$

$$
c^{*}=\sup _{c>0}\left\{c \mid \inf _{V \in \mathcal{X}} E_{c}(V)<0\right\},
$$

where $\mathcal{X}:=\left\{V \in\left[H_{\mathrm{loc}}^{1}(\mathbb{R})\right]^{N}: V( \pm \infty)=a^{ \pm}\right\}$.

We now derive a priori bounds on $c^{*}$. We take $t>0$ and consider the affine $\left[W_{\text {loc }}^{1, \infty}(\mathbb{R})\right]^{N}$ function

$$
U_{\text {aff }}^{t}(x):=a^{-} \chi_{(-\infty,-t)}+\left(\frac{t-x}{2 t} a^{-}+\frac{t+x}{2 t} a^{+}\right) \chi_{[-t, t]}+a^{+} \chi_{(t, \infty)} .
$$

Proposition 34 (A priori bounds on $c^{*}$ ). There exist $0<c_{\min }<c_{\max }<\infty$ depending only on $W$, such that

$$
c_{\min } \leq c^{*} \leq c_{\max } .
$$

Moreover, if $d_{0}:=\lim _{\alpha \rightarrow 0^{+}} d_{\alpha}$, then

$$
\begin{aligned}
c_{\max } & =\frac{\sqrt{2 W^{-}\left(a^{-}\right)}}{d_{0}} \\
c_{\min } & =\sup _{t>0}\left[\frac{W^{-}\left(a^{-}\right)}{e^{2 t c_{\max }}}\left(\frac{1}{2}\left\{\frac{\left|a^{+}-a^{-}\right|}{2 t}\right\}^{2}+\int_{-t}^{t} W^{+}\left(\frac{t-x}{2 t} a^{-}+\frac{t+x}{2 t} a^{+}\right) d x\right)^{-1}\right] .
\end{aligned}
$$

Proof of Proposition 34. The upper bound follows by Lemmas 27 and 29, For the lower bound, we utilize (30) and take as we can $t=L$. This gives as in (5) that the inequality $0=E_{c}\left(U_{t}\right) \leq E_{c}\left(U_{\text {aff }}^{t}\right)$ implies

$$
0 \leq-e^{-c t} \frac{W^{-}\left(a^{-}\right)}{c}+e^{c t} \int_{-t}^{t}\left\{\frac{1}{2}\left|\frac{a^{+}-a^{-}}{2 t}\right|^{2}+W^{+}\left(\frac{t-x}{2 t} a^{-}+\frac{t+x}{2 t} a^{+}\right)\right\} d x .
$$

Hence, for all $t>0$,

$$
c \geq \frac{W^{-}\left(a^{-}\right)}{e^{2 c t}}\left(\int_{-t}^{t}\left\{\frac{1}{2}\left|\frac{a^{+}-a^{-}}{2 t}\right|^{2}+W^{+}\left(\frac{t-x}{2 t} a^{-}+\frac{t+x}{2 t} a^{+}\right)\right\} d x\right)^{-1} .
$$

Utilizing the upper bound and maximizing with respect to $t>0$, we are done.

\section{Extensions}

Utilizing ideas related to those in $\underline{\mathrm{A}-\mathrm{F}}$, we relax $\left(\mathrm{h}^{*}\right)$ to a localized version. The new (h*) requires the existence of two convex components $\mathcal{C}_{\alpha}^{ \pm}$of the sublevel set $\{W \leq \alpha\}$, but only when $W$ is restricted in a large convex set $\Omega \subseteq \mathbb{R}^{N}$ without any restriction on $\left.W\right|_{\operatorname{ext}(\Omega)}$. As a consequence, $\left(\mathrm{h}^{* *}\right.$ allows for potentials with several other minima and/or unbounded values to $-\infty$.

There exists a $C^{2}$ convex closed set $\Omega \subseteq \mathbb{R}^{N}$ which encloses the minima $\left(\mathrm{h}^{* *}\right) \quad a^{ \pm}$and satisfies (H3), such that (h*) holds for $W$ within $\Omega$. Moreover, the values of $W$ on $\partial \Omega$ exceed those in the interior : if $u \in \operatorname{int}(\Omega)$, then $W(u)<\min _{\partial \Omega} W$.

\footnotetext{
${ }^{3}$ Analogous characterizations have been obtained in [H-P-S] and [He] for other travelling wave problems.
} 
Example 35 (N-d potentials satisfying ( of the 2-well potential $W(u):=\left|u-a^{+}\right|^{p}\left|u-a^{-}\right|^{p}, p \geq 2, u \in \mathbb{R}^{N}$. We take $\varepsilon>0$ and set

$$
F_{\varepsilon}(u):=\left\{\varepsilon \exp \left(\left(\left|u-a^{-}\right|^{2}-\delta^{2}\right)^{-1}\right)+1\right\} \chi_{\mathbb{B}\left(a^{-}, \delta\right)}+\chi_{\mathbb{R}^{N} \backslash \mathbb{B}\left(a^{-}, \delta\right)},
$$

where $C:=\max _{\left|u-a^{-}\right|=\delta}\{W(u)\}$ and define $W_{\varepsilon}(u):=F_{\varepsilon}(u) W(u)-C\left(F_{\varepsilon}(u)-1\right)$. The potentials $W_{\varepsilon}$ satisfy our assumptions and $W_{\varepsilon}-W \longrightarrow 0$ in $C^{2}\left(\mathbb{R}^{N}\right)$, as $\varepsilon \rightarrow 0^{+}$.

(ii) (G. Paschalides) The following deformation of the 2-well planar potential,

$$
W_{C}\left(u_{1}, u_{2}\right):= \begin{cases}W\left(u_{1}, u_{2}\right), & u_{1}<0, u_{2} \in \mathbb{R}, \\ W\left(u_{1}, u_{2}\right)-C\left[6 u_{1}^{5}-15 u_{1}^{4}+10 u_{1}^{3}\right], & 0 \leq u_{1} \leq 1, u_{2} \in \mathbb{R}, \\ W\left(u_{1}, u_{2}\right)-C, & u_{1}>1, u_{2} \in \mathbb{R},\end{cases}
$$

(with $a^{ \pm}=( \pm 1,0)$ ) satisfies the assumptions ( $\left(\mathrm{h}^{*}\right),\left(\mathrm{h}^{* *}\right)$ for any $C>0$.

Remark 36. Can monotonicity of (h*), (h**) be relaxed? In the Appendix we construct a class of $W$ 's which are monotone except for merely one critical point $a^{0}$ in $W^{-1}\left(\left[W\left(a^{-}\right), 0\right]\right)$. This implies the existence of a connection $a^{+}-a^{0}$, different from $a^{+}-a^{-}$, which generally obstructs existence. Critical points at a lower level attract, for $c>0$, the flow of $U_{x x}-\nabla W(U)=-c U_{x}$ (see also Risler $\underline{\mathrm{R}}$ ).

Extension of Theorem 32 under the assumption $\left(\mathbf{h}^{* *}\right)$. In this case we solve a related problem for a modified "better" $\bar{W}$ and then show that the solution we construct is also a solution of the original problem as well. We modify $W$ to a new $\bar{W}$ by setting:

$$
\bar{W}:=W \chi_{\left\{W \geq \min _{\partial \Omega} W\right\}}+\left(2 \min _{\partial \Omega} W-W\right) \chi_{\left\{W<\min _{\partial \Omega} W\right\}} .
$$

This is the reflection of the graph of $W$ with respect to the hyperplane $\{w=$ $\left.\min _{\partial \Omega} W\right\}$ which maps any parts of $G r(W)$ lying into $\left\{W \leq \min _{\partial \Omega} W\right\}$ to the opposite halfspace. $\bar{W}$ is sufficiently coercive and Lemma 12 applied to $\Omega$ and to $E_{c}$ provides an $\left[L^{\infty}(\mathbb{R})\right]^{N}$-bound for the minimizers, showing that they are localized inside $\Omega$. Since $\bar{W}$ satisfies ( $\left.\mathrm{h}^{*}\right)$ inside $\Omega$, problem (1) for $\bar{W}$ has a solution $U$ in $\left[C^{2}(\mathbb{R})\right]^{N}$. By construction $\left.\left.W\right|_{\Omega} \equiv \bar{W}\right|_{\Omega}$, so $U$ solves (1) for $W$ as well.

\section{Appendix}

On the optimality of the assumptions. We construct a class of $W$ 's for which there is a heteroclinic map between a local minimum $a^{+}$with $W\left(a^{+}\right)=0$ and a critical point $a^{0}$ with $0>W\left(a^{0}\right)>W\left(a^{-}\right), a^{-}$the global minimum. Hence, the existence of additional solutions which may obstruct the existence of $a^{+}-a^{-}$ connections cannot be excluded without monotonicity as in (h*).

$$
\text { We assume that } W \in C_{\mathrm{loc}}^{2}\left(\mathbb{R}^{N}\right) \text { and }
$$

(1) $W$ has at least 3 critical points, $a^{ \pm}, a^{0}$ with $a^{ \pm}$local minima, $a^{0}$ a critical point and $W\left(a^{+}\right)=0>W\left(a^{0}\right)>W\left(a^{-}\right)$.

(2) For $2 \leq j \leq N, W_{u_{j}}\left(u_{1}, 0, \ldots, 0\right)=0$ and $\left[a^{-}, a^{0}\right],\left[a^{0}, a^{+}\right]$are on the $u_{1}$-axis.

If $N=1$ and $a^{0}$ is a local minimum, then generally no $a^{+}-a^{-}$connection exists ([F-McL $)$, depending on the speeds $c_{-, 0}$ and $c_{0,+}$ of the solutions $a^{-}-a^{0}$ and $a^{0}-a^{+}$. For $N>1,(b)$ implies the existence of solutions $U=(u, 0, \ldots, 0)$ to 


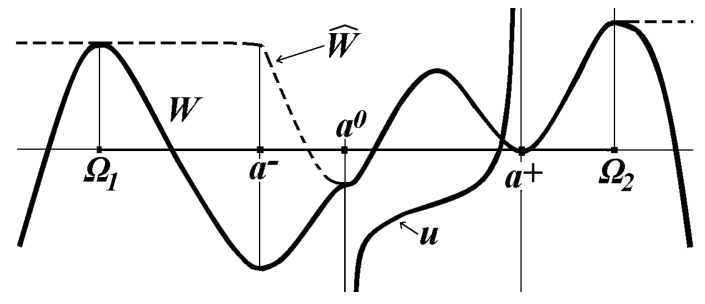

$U_{x x}-\nabla W(U)=-c U_{x}$ for the slice $\bar{W}(u):=W(u, 0, \ldots, 0)$. Thus, we may only impose assumptions on $\bar{W}$ :

We assume that (h**) holds, with the exception that $\bar{W}$ is monotone on $\left(a^{-}, a^{0}\right)\left(a^{0}, a^{+}\right)$separately, instead of $\left(a^{-}, a^{+}\right)$.

Proposition. If $W$ satisfies (h1), (h2), there exists a solution $(U, c) \in\left[C^{2}(\mathbb{R})\right]^{N} \times$ $(0, \infty)$ to

$$
\left\{\begin{array}{l}
U_{x x}-\nabla W(U)=-c U_{x} \\
U(+\infty)=a^{+}, U(-\infty)=a^{0}
\end{array}\right.
$$

Proof of Proposition. We deform smoothly the slice $\bar{W}$ to a new $\widehat{W}$ for which the nature of the critical point $a^{0}$ is changed, being a global minimum of $\widehat{W}$. Then, the problem for $\widehat{W}$ can be tackled by the foregoing theory, and, by a localization argument, the solution we construct also solves the original problem. Let $F:\left(a^{-}, a^{0}\right) \longrightarrow(0, \infty)$ be the "half" of the standard bell function $F(u):=K \exp \left(\left(u-a^{0}\right)^{-1}\left(u-a^{0}+2 a^{-}\right)^{-1}\right), K>0$ to be chosen, and consider the following transformation:

$$
\widehat{W}(u):= \begin{cases}\bar{W}\left(\Omega_{2}\right), & u \geq \Omega_{2}, \\ \bar{W}(u), & u \in\left[a^{0}, \Omega_{2}\right), \\ -\left(F(u) \bar{W}(u)-2 \bar{W}\left(a^{-}\right)\right), & u \in\left(a^{-}, a^{0}\right), \\ -\left(F\left(a^{-}\right) \bar{W}(u)-2 \bar{W}\left(a^{-}\right)\right), & u \leq a^{-} .\end{cases}
$$

We choose $K>0$, such that $\widehat{W}\left(a^{-}\right) \geq \bar{W}\left(\Omega_{1}\right)$. Assumptions (h1), h2) imply that $\widehat{W}$ satisfies (h*), giving an $a^{+}-a^{0}$ heteroclinic function which solves $u_{x x}-\widehat{W}^{\prime}(u)=$ $-c u_{x}$ (Theorem 32). Lemma 12 provides the $L^{\infty}(\mathbb{R})$-bound

$$
a^{-} \leq u(x) \leq \Omega_{2}, \quad \text { for all } x \in \mathbb{R} .
$$

The function $u$ solves $u_{x x}-\bar{W}^{\prime}(u)=-c u_{x}$ as well. Indeed, it suffices to improve the bound on $u$ to $a^{0} \leq u(x) \leq a^{+}$, for all $x \in \mathbb{R}$. Since by construction $\left.\bar{W}\right|_{\left[a^{0}, a^{+}\right]} \equiv$ $\left.\widehat{W}\right|_{\left[a^{0}, a^{+}\right]}$, Lemma 12 applied to (3) for $\widehat{W}$ gives the desired localization.

\section{ACKNOWLEDGEMENT}

We thank Peter Bates, Vassilis Papanicolaou and Achilleas Tertikas for their suggestions and their interest in the present work. We also thank Gregory Paschalides for Example 35] and the numerical simulation. Special thanks are due to the anonymous referee for his several suggestions and valuable comments which improved the 
content as well as the presentation of this paper. Finally, we wish to thank Hiroshi Matano for the information he gave us on the status of the problem.

\section{REFERENCES}

[A-Ba-C] N. Alikakos, P. Bates, X. Chen, Periodic travelling waves and oscillating patterns in multidimensional domains, Transactions of the A.M.S., Vol. 351, Nr. 7, (1999), 27772805. MR $1467460(99 \mathrm{j}: 35101)$

[A-Be-C] N. Alikakos, S. Betelú, X. Chen, Explicit Stationary Solutions in Multiple Well Dynamics and Non-uniqueness of Interfacial Energy Densities, Euro. Jnl. of Applied Math. (2006), 17, 525-556. MR 2296027 (2007k:35119)

[A-F] N. Alikakos, G. Fusco, On the connection problem for potentials with several global minima, Indiana Univ. J. of Math., Vol. 57, No. 4, 1871 - 1906, (2008). MR2440884 (2009h:37124)

[C et al.] X. Chen, J.-S. Guo, F. Hamel, H. Ninomiya, J.-M. Roquejoffre, Traveling waves with paraboloid like interfaces for balanced bistable dynamics, Ann. Inst. H. Poincaré Anal. Non Linéaire 24 (2007), 369-393. MR2319939 (2009g:35063)

[DC] M. P. Do Carmo, Differential Geometry of Curves and Surfaces, Prentice-Hall, 1976 (25th printing). MR0394451 (52:15253)

[Ev] L. C. Evans, Partial Differential Equations, A.M.S., Graduate Texts in Mathematics, Vol. 19, 1998. MR1625845 (99e:35001)

[F] P. Fife, Long time behavior of solutions of bistable nonlinear diffusion equations, Arch. Rat. Mech. Anal. 70 (1979), 31-46. MR 535630 (81f:35063)

[F-McL] P. Fife, J. B. McLeod, The approach of solutions of nonlinear diffusion equations to travelling front solutions, Arch. Rat. Mech. Anal. 65 (1977), 335-361. MR0442480 $(56: 862)$

[F-McL2] P. Fife, J. B. McLeod, A phase plane discussion of convergence to travelling fronts for nonlinear diffusion, Arch. Rat. Mech. Anal. 75 (1981), 281-314. MR607901(83b:35085)

[G-R] T. Gallay, E. Risler, A variational proof of global stability for bistable travelling waves, Diff. and Int. Equations 20 (2007), 901-926. MR2339843 (2008m:35196)

[G-H-L] S. Gallot, D. Hulin, J. Lafontaine, Riemannian Geometry, Springer-Verlag, 1993, 2nd printing.

[G-T] D. Gilbarg, N. Trudinger, Elliptic Partial Differenial Equations of Second Order, Springer, 1998, revised 3rd edition.

[Hei] S. Heinze, Travelling Waves for Semilinear Parabolic Partial Differential Equations in Cylindrical Domains, Ph.D. thesis, Heidelberg University, 1988.

[H-P-S] S. Heinze, G. Papanicolaou, A. Stevens, Variational principles for propagation speeds in inhomogeneous media, SIAM J. of Appl. Math. (2001), Vol. 62, No. 1, 129-148. MR:1857539(2002j:35169)

[He] D. Henry, Geometric Theory of Semilinear Parabolic Equations, Lecture Notes in Mathematics 840, Springer-Verlag, 1981. MR610244 (83j:35084)

[K-S] A. Kufner, A.-M. Sändig, Some Application of Weighted Sobolev Spaces, Leipzig, Teubner-Texte zur Mathematik, 1987. MR.926688 (89h:35096)

[LMN] M. Lucia, C. Muratov and M. Novaga, Existence of traveling wave solutions for Ginzburg-Landau-type problems in infinite cylinders, Arch. Rat. Mech. Anal., vol. 188, n 3, 475-508, 2008. MR2393438 (2009i:35181)

[M] C. B. Muratov, A global variational structure and propagation of disturbances in reaction-diffusion systems of gradient type, Disc. Cont. Dyn. Syst. Ser B 4 (2004), 867-892. MR.2082914 (2005j:35124)

[R] E. Risler, Global convergence towards travelling fronts in nonlinear parabolic systems with a gradient structure, Annales de l'Institut Henri Poincaré (C) Anal. Non Linéaire Analysis, Vol. 25, Issue 2, 381-424 (2008). MR2400108 (2009b:35202)

[Stef] V. Stefanopoulos, Heteroclinic connections for multiple-well potentials: The anisotropic case, Proceedings of the Royal Society of Edinburgh, 138A, 13131330, 2008. MR 2488061 (2009m:37058)

[St] P. Sternberg, Vector-valued local minimizers of nonconvex variational problems, Rocky Mountain J. of Math., 21, (1991), no. 2, 799-807. MR.1121542(92e:49016) 
[V] A. Volpert, V. Volpert, V. Volpert, Traveling wave solutions of parabolic systems, A.M.S., Translations of Mathematical Monographs Vol. 140, 1994. MR.1297766 (96c:35092)

Department of Mathematics, University of Athens, Panepistimioupolis 11584, Athens, Greece - And - Institute of Applied and Computational Mathematics, Foundation for Research and Technology, GR 70013 Heraklion, Crete, Greece

E-mail address: nalikako@math.uoa.gr

Department of Mathematics, University of Athens, Panepistimioupolis 11584, Athens, Greece

E-mail address: nkatzourakis@math.uoa.gr 